\title{
Numerical pricing of American options under two stochastic factor models with jumps using a meshless local Petrov-Galerkin method
}

\author{
Jamal Amani Rad ${ }^{\mathrm{a}, \mathrm{b}, *}$, Kourosh Parand ${ }^{\mathrm{a}, \mathrm{b}}$ \\ ${ }^{a}$ Department of Computer Sciences, Faculty of Mathematical Sciences, Shahid Beheshti University, G.C. Tehran, Iran \\ ${ }^{b}$ Department of Cognitive Modeling, Institute for Cognitive and Brain Sciences, Shahid Beheshti University, G.C. Tehran, \\ Iran
}

\begin{abstract}
The most recent update of financial option models is American options under stochastic volatility models with jumps in returns (SVJ) and stochastic volatility models with jumps in returns and volatility (SVCJ). To evaluate these options, mesh-based methods are applied in a number of papers but it is well-known that these methods depend strongly on the mesh properties which is the major disadvantage of them. Therefore, we propose the use of the meshless methods to solve the aforementioned options models, especially in this work we select and analyze one scheme of them, named local radial point interpolation (LRPI) based on Wendland's compactly supported radial basis functions (WCS-RBFs) with $C^{6}, C^{4}$ and $C^{2}$ smoothness degrees. The LRPI method which is a special type of meshless local Petrov-Galerkin method (MLPG), offers several advantages over the mesh-based methods, nevertheless it has never been applied to option pricing, at least to the very best of our knowledge. These schemes are the truly meshless methods, because, a traditional non-overlapping continuous mesh is not required, neither for the construction of the shape functions, nor for the integration of the local sub-domains. In this work, the American option which is a free boundary problem, is reduced to a problem with fixed boundary using a Richardson extrapolation technique. Then the implicit-explicit (IMEX) time stepping scheme is employed for the time derivative. Numerical experiments are presented showing that the proposed approaches are extremely accurate and fast.
\end{abstract}

Keywords: Stochastic volatility, American option, Merton jump diffusion, Meshless weak form, Wendland functions.

AMS subject classification: 91G80; 91G60; 35R35.

\section{Introduction}

In the field of numerical methods, the meshless methods have attracted considerable interest over the past decade. The main advantage of these methods is to approximate the unknown field by a linear combination of shape functions built without having recourse to a mesh of the domain. In this method, we use a set of nodes scattered within the domain of the problem as well as sets of nodes on the boundaries of the domain to represent (not discretize) the domain of the problem and its boundaries [22]. These sets of scattered nodes do not form a mesh, meaning it does not require any priori information on the relation ship between the nodes for the interpolation or approximation of the unknown functions of field variables [42].

Meshless methods have progressed remarkably in the last decades. They are mainly based on the weak from representation (see [58, 2, 1, 25, 23]) and the strong form based on the collocation techniques (see $[47,50,4,5])$, although a combination of both approaches is possible [41]. One limitation of the collocation methods is less accuracy and lower stability in numerical implementation. On the other hands, the weak forms are used to derive a set of algebraic equations through a numerical integration process using a set of quadrature domain that may be constructed globally or locally in the domain of the problem, such as the meshless local Petrov-Galerkin method (MLPG) based on local weak-form and the radial basis functions

\footnotetext{
${ }^{*}$ Corresponding author

Email addresses: j.amanirad@gmail.com;j_amanirad@sbu.ac.ir (Jamal Amani Rad), k_parand@sbu.ac.ir (Kourosh Parand)
}

Preprint submitted to .

January 18, 2017

(C) 2017. This manuscript version is made available under the Elsevier user license

http://www.elsevier.com/open-access/userlicense/1.0/ 
(RBFs) approximation developed by Atluri and his colleagues [7]. This method also known as local radial point interpolation (LRPI) method. The MLPG method is a truly meshless method, because, a traditional non-overlapping, continuous mesh is not required, either for the construction of the shape function, or for the integration of the local sub-domain. The trial and test function spaces can be different or the same. Particularly, the LRPI meshless method reduces the problem dimension by one, has shape functions with delta function properties, and expresses the derivatives of shape functions explicitly and readily. Thus it allows one to easily impose essential boundary and initial (or final) conditions. The method has now been successfully extended to a wide range of problems in engineering. For some examples of these problems, see $[22,24]$ and other references therein. The interested reader of meshless methods can also see $[58,2,1]$.

Over the last years, the growth of the financial markets has been an ever expanding economical field. In all over the world, the value of the financial assets traded on the stock markets has reached astronomical amounts. Trading of financial derivatives such as options is a continuous business going on all over the world. Making sure that the price are correct at every time is of great importance for the traders.

Options are financial contracts that gives to the buyer the right, to buy (call option) or to sell (put option) an underlying asset (such as a stock) at a previous agreed price. Called the strike or exercise price on or before a certain time called the maturity. Mast of these options can be grouped into either of the two categories: European options which can only be exercised at one given expiry or maturity date $(t=T)$ and American options can additionally be executed at any time prior to their maturity date $(t \leq T)$. The American options give the freedom when to use the option and are often a little bit more expensive than a corresponding European options.

Options are priced using mathematical models that are often challenging to solve $[53,52,51,49]$. In particular, the famous Black-Scholes model [13] yields explicit pricing formulae for some kinds of European options, including vanilla call and put. Recently, by using very empirical studies, it has become evident that the assumption of behavior like a log-normal asset value diffusion with a constant volatility and a drift in the standard Black-Scholes model of the underlying asset price is not consistent with that the real market prices of options with various strike prices and maturities such as volatility smile or skew and heavy tails $[3,56]$.

During the last decade, many works have been done to find modifications of classical Black-Scholes model to satisfy these phenomena in financial markets such as the models with stochastic volatility (SV), the models with jumps (such as Merton and Kou models proposed by Merton and Kou in two different works [44] and [37], respectively), their combinations of stochastic volatility and jumps in returns, i.e. stochastic volatility models with jumps (SVJ) introduced by Bates [12], and stochastic volatility models with jumps in returns and volatility (SVCJ) introduced by Duffie et al. [26].

In this research, we focus on the SVJ and SVCJ models. Note that Merton model is selected to jump term of model here. The mentioned SVJ and SVCJ models for pricing American options are governed by a parabolic integro-differential variational inequality which can be formulated as a free boundary problem. In particular, these models are contain differential term and a nonlocal integral term. Moreover it should be noted that in this model an analytical solution is impossible because of the early exercise possibility of American options. Therefore, to solve these problems, we need to have a powerful computational method. To this aim, several numerical methods have been proposed for pricing options under SVJ and SVCJ models (see, e.g., $[11,55,57,17,60]$ ) but weak form meshless methods have never been used for option pricing of this model.

The objective of this paper is to extend the LRPI based on Wendland's compactly supported radial basis functions (WCS-RBFs) with $C^{6}, C^{4}$ and $C^{2}$ smoothness [61] to evaluate American options under SVJ and SVCJ models. Again we do emphasize that the local weak form of meshless method has not yet been used in mathematical finance. Therefore, it appears to be interesting to extend such a numerical technique also to option valuation, which is done in the presented manuscript.

In addition, in this paper the infinite space domain $\mathbb{R}^{+} \times \mathbb{R}^{+}$is truncated to $\left[0, S_{\max }\right] \times\left[0, y_{\max }\right]$ in SVJ and SVCJ models, with the sufficiently large values $S_{\max }$ and $y_{\max }$ to avoid an unacceptably large truncation error. The options' payoffs considered in this paper are non-smooth functions, in particular their derivatives are discontinuous at the strike price. Therefore, to reduce as much as possible the losses of accuracy the points of the trial functions are concentrated in a spatial region close to the strike prices. So, we employ the change of variables proposed by Clarke and Parrott [20].

As far as the time discretization is concerned, we use the implicit-explicit (IMEX) time stepping scheme. 
Note that in SVJ and SVCJ models, the integral part is a non-local integral, whereas the other parts which are differential operators, are all local. No doubt, since the integral part is non-local operator, a dense linear system of equations will be obtained by using the $\theta$-weighted discretization scheme. Therefore, to obtain a sparse linear system of equations, it is better to use a IMEX scheme which is noted for avoiding dense matrices. So far published work existing in the literature which use the IMEX scheme to price the options, include [11, 57, 28, 39, 40]. Such an approach can be first-order accurate like [11, 28] or second-order accurate like $[39,40]$. In this work we use the first-order type of IMEX schemes, then a second-order time discretization is obtained by performing a Richardson extrapolation procedure with halved time step.

Finally, in order to solve the free boundary problem that arises in the case of American options is computed by Richardson extrapolation of the price of Bermudan option. In essence the Richardson extrapolation reduces the free boundary problem and linear complementarity problem (LCP) to a fixed boundary problem which is much simpler to solve.

Numerical experiments are presented showing that the proposed approach is very efficient from the computational standpoint. In particular, the prices of both European and American options in SVJ or SVCJ models can be computed with an error (in both the maximum norm and the root mean square relative difference) of order $10^{-3}$ in few tenth of a second. Moreover, the Bermudan approximation reveals to be the most efficient of the algorithms used to deal with the early exercise opportunity.

We remark that the main contribution of this manuscript is to show that the MLPG, which has never been applied to problems in mathematical finance, can yield accurate and fast approximations of European and American option prices.

Overall, our focus in this paper is more devoted to providing an accurate, computationally fast, stable, convergence and simple technique for pricing options under SVJ and SVCJ models. We should note that the key idea of this work is finding a new technique combined using the following numerical tools : Spatial change of variables, time discretization of the integro-differential operator, Richardson extrapolation procedure, LRPI discretization, Wendland's compactly supported radial basis functions, Spatial variable transformation, approximate the price of the American option with the price of a Bermudan option and $L U$ factorization method with partial pivoting. Rigorously speaking, these tools considered separately, are not new, but here due to the fact that an approach that puts all these techniques together has never been proposed in option pricing, therefore the proposed technique is new, accurate and very fast.

The paper is organized as follows: In Section 2 a detailed description of the SVJ and SVCJ models for American options is provided. Section 3 is devoted to presenting the MLPG approach and the application of such a numerical technique to the option pricing problems considered is shown in this section. The numerical results obtained are presented and discussed in Section 4 and finally, in Section 5, some conclusions are drawn.

\section{The option pricing models}

\subsection{SVJ or Bates SV model}

For the sake of simplicity, from now we restrict our attention to options of call type, but the case of put options can be treated in perfect analogy.

First we are interested in pricing a American call option of the Bates stochastic volatility model which is the exponential Lévy processes consisting of a two-dimensional geometric Brownian motion plus a compound Possion jumps with time varying volatility.

We now consider the pricing of an American call option denoted by $V(s, y, t)$ on the underlying asset $S_{t}$ and its volatility $Y_{t}$ with asset price $E$ and maturity $T$. On can show that $V(s, y, t)$, for $s, y \in[0,+\infty)$ and $t \in[0, T)$, satisfy the following system of free boundary problem $[11,17]$

$$
\begin{array}{lr}
\frac{\partial}{\partial t} V(s, y, t)+\mathcal{L} V(s, y, t)=0, & 0 \leq s<B(y, t), \\
V(s, y, t)=s-E, & s>B(y, t),
\end{array}
$$


with exercise boundary conditions as follow $[11,17]$

$$
\begin{aligned}
& \lim _{s \rightarrow B(y, t)} \frac{\partial V(s, y, t)}{\partial s}=1, \\
& \lim _{s \rightarrow B(y, t)} \frac{\partial V(s, y, t)}{\partial y}=0,
\end{aligned}
$$

where

$$
\begin{gathered}
\mathcal{L} V(s, y, t)=-\mathbf{F} \nabla V(s, y, t)+\nabla \cdot(\mathbf{E} \nabla V(s, y, t))-(r+\lambda) V(s, y, t) \\
+\lambda \int_{\mathbb{R}^{+}} V(s x, y, t) f(x) d x, \\
\mathbf{E}=\frac{1}{2}\left(\begin{array}{cc}
y s^{2} & \rho \theta s y \\
\rho \theta s y & \theta^{2} y
\end{array}\right), \\
\mathbf{F}=-\left(\begin{array}{c}
(r-q-\lambda \kappa) s-y s-\rho \frac{\theta}{2} s \\
\xi(\eta-y)-\frac{\theta^{2}}{2}-\rho \frac{\theta}{2} y
\end{array}\right)^{T},
\end{gathered}
$$

with $\rho \in[-1,1]$ being the the correlation factor, $\lambda$ being the intensity of Possion process and $\alpha=r-q-\lambda \kappa$ being the drift rate, where $r$ is the risk-free interest rate, $q$ is the dividend and $\kappa$ is the expected relative jump size. Moreover, $f(x)$ is a weight function in the Lévy measure. By selecting this weight function the finite activity jump-diffusion model is the log-normal model proposed by Merton [44]

$$
f(x)=\frac{1}{\sqrt{2 \pi} x \delta} \exp \left(-\frac{(\log x-\gamma)^{2}}{2 \delta^{2}}\right)
$$

Note that $f(x) \geq 0$ and

$$
\int_{\mathbb{R}^{+}} f(x) d x=1
$$

Again we should note that $\kappa$ is the expected relative jump size and is computed as $\kappa=\int_{\mathbb{R}^{+}}(z-1) f(z) d z$. We have $\kappa=\exp \left(\gamma+\delta^{2} / 2\right)-1$ for Merton model.

The value of $V$ at maturity is given by

$$
V(s, y, T)=\varsigma(s)
$$

where $\varsigma$ is the so-called option's payoff:

$$
\varsigma(s)=\max (s-E, 0),
$$

which is clearly not differentiable at $s=E$.

The behavior of the value of the American call option on the boundaries is given by

$$
V(0, y, t)=0, \quad \lim _{s \rightarrow+\infty} V(s, y, t)=s-E .
$$

In relations (2.1)-(2.4), $B(y, t)$ denotes the so-called exercise boundary, which is unknown and is implicitly defined by (2.1)-(2.10). The above free-boundary partial differential problem does not have an exact closedform solution, and thus some numerical approximation is required.

Problem (2.1)-(2.10) can be reformulated as a linear complementarity problem:

$$
\begin{aligned}
& \frac{\partial}{\partial t} V(s, y, t)+\mathcal{L} V(s, y, t) \geq 0, \\
& V(s, y, t)-\varsigma(s) \geq 0, \\
& \left(\frac{\partial}{\partial t} V(s, y, t)+\mathcal{L} V(s, y, t)\right) \cdot(V(s, y, t)-\varsigma(s))=0,
\end{aligned}
$$


which holds for $s, y \in(0,+\infty)$ and $t \in[0, T)$, with final condition:

$$
V(s, y, T)=\varsigma(s),
$$

and boundary conditions:

$$
V(0, y, t)=0, \quad \lim _{s \rightarrow+\infty} V(s, y, t)=s-E .
$$

\subsection{SVCJ model}

In the Bates models, Poisson jumps are only added to the risk-neutral dynamic of the asset price $S_{t}$. In contrast, in the SVCJ models, jumps are appeared in the volatility, too. Let $V(s, y, t)$ denote the price of a American derivative on an underlying asset described by stochastic models mentioned in [57, 26]. As in $[57,28,26]$, it can be shown that $V(s, y, t)$ is governed by the partial integro-differential equation

$$
\begin{array}{lr}
\frac{\partial}{\partial t} V(s, y, t)+\mathcal{L} V(s, y, t)=0, & 0 \leq s<B(y, t), \\
V(s, y, t)=s-E, & s>B(y, t),
\end{array}
$$

where

$$
\begin{aligned}
& \mathcal{L} V(s, y, t)=-\mathbf{F} \nabla V(s, y, t)+\nabla \cdot(\mathbf{E} \nabla V(s, y, t))-(r+\lambda) V(s, y, t) \\
& +\lambda \int_{\mathbb{R}^{+}} \int_{\mathbb{R}^{+}} V\left(s z^{s}, y+z^{v}, t\right) f\left(z^{s}, z^{v}\right) d z^{v} d z^{s}, \\
& \mathbf{E}=\frac{1}{2}\left(\begin{array}{cc}
y s^{2} & \rho \theta s y \\
\rho \theta s y & \theta^{2} y
\end{array}\right) \\
& \mathbf{F}=-\left(\begin{array}{c}
(r-q-\lambda \kappa) s-y s-\rho \frac{\theta}{2} s \\
\xi(\eta-y)-\frac{\theta^{2}}{2}-\rho \frac{\theta}{2} y
\end{array}\right)^{T},
\end{aligned}
$$

the final and boundary conditions for this model are described in relations (2.14) and (2.15). Moreover $\rho$ defines the correlation between jumps in returns and variance, $\lambda$ is the intensity in compound Poisson process $[57,26]$. The distribution of the jump size in variance is assumed to be exponential with mean $\nu$. Conditional on a jump of size $z^{v}$ in the variance process, we have a $\log$-normal distribution $f\left(z^{s}, z^{v}\right)$ with the mean in $\log z^{s}$ being $\gamma+\rho z^{v}$ [57]. This gives a bivariate probability density function defined by [57]

$$
f\left(z^{s}, z^{v}\right)=\frac{1}{\sqrt{2 \pi} z^{s} \delta \nu} \exp \left(-\frac{z^{v}}{\nu}-\frac{\left(\log z^{s}-\gamma-\rho_{j} z^{v}\right)^{2}}{2 \delta^{2}}\right) .
$$

\section{Methodology}

In this work, the price of American option is computed by Richardson extrapolation of the price of Bermudan option. Richardson extrapolation method has been successfully extended to a wide range of option pricing problem. For some examples of these problems, see $[18,28,11,10]$. In essence the Richardson extrapolation reduces the free boundary problem and linear complementarity problem to a fixed boundary problem which is much simpler to solve. Moreover Richardson extrapolation is computationally faster than LCP or the penalty methods used to price American options, see [11, 53]. Thus, instead of describing the aforementioned linear complementarity problem or penalty method, we directly focus our attention onto the partial integro-differential equation satisfied by the price of a Bermudan option which is faster and more accurate than other methods.

For the sake of simplicity exposition, we restrict our attention to option of the Bates stochastic volatility model, but the case of options under SVCJ model can be treated in perfect analogy. 
Let us consider in the interval $[0, T], M+1$ equally spaced time levels $t_{0}=0, t_{1}, t_{2}, \ldots, t_{M}=T$. Let $V_{M}(s, y, t)$ denote the price of a Bermudan option with maturity $T$ and strike price $E$. The Bermudan option is an option that can be exercised not on the whole time interval $[0, T]$, but only at the dates $t_{0}, t_{1}$, $\ldots, t_{M}$. That is we consider the problems

$$
\left\{\begin{array}{l}
\frac{\partial}{\partial t} V_{M}(s, y, t)+\mathcal{L} V_{M}(s, y, t)=0, \\
V_{M}(0, y, t)=0, \quad \lim _{s \rightarrow+\infty} V_{M}(s, y, t)=s-E .
\end{array}\right.
$$

which hold in the time intervals $\left(t_{0}, t_{1}\right),\left(t_{1}, t_{2}\right), \ldots,\left(t_{M-1}, t_{M}\right)$. By doing that also the relation (2.13) is automatically satisfied in every time interval $\left(t_{k}, t_{k+1}\right), k=0,1, \ldots, M-1$. Moreover, the relation (2.12) is enforced only at times $t_{0}, t_{1}, \ldots, t_{M-1}$, by setting

$$
V_{M}\left(s, y, t_{k}\right)=\max \left(\lim _{t \rightarrow t_{k}^{+}} V_{M}(s, y, t), \varsigma(s)\right), \quad k=0,1, \ldots, M-1 .
$$

Note that the function $V_{M}\left(\cdot, \cdot, t_{k}\right)$ computed according to (3.2) is used as the final condition for the problem (3.1) that holds in the time interval $\left(t_{k-1}, t_{k}\right), k=1,2, \ldots, M-1$. Instead, the final condition for the problem (3.1) that holds in the time interval $\left(t_{M-1}, t_{M}\right)$, according to the relation (2.14), is prescribed as follows:

$$
V_{M}\left(s, y, t_{M}\right)=\varsigma(s) .
$$

That is, in summary, problems (3.1) are recursively solved for $k=M-1, M-2, \ldots, 0$, starting from the condition (3.3), and at each time $t_{M-1}, t_{M-2}, \ldots, t_{0}$ the American constraint (3.2) is imposed.

The Bermudan option price $V_{M}(s, y, t)$ tends to become a fair approximation of the American option price $V(s, y, t)$ as the number of exercise dates $M$ increases. In this work the accuracy of $V_{M}(s, y, t)$ is enhanced by Richardson extrapolation which is second-order accurate in time.

To evaluate the option, the meshless local Petrov-Galerkin (MLPG) method is used in this work. This method is based on local weak forms over intersecting sub-domains, which are extracted over the local sub-domains using divergence theorem and a indicator test function. At first we discuss a time-stepping method for the time derivative.

\subsection{Time discretization}

First of all, we discretize the operator (2.5) in time. For this propose, we can apply the Laplace transform or use a time-stepping approximation. Algorithms for the numerical inversion of a Laplace transform lead to a reduction in accuracy. Then, we employ a time-stepping method to overcome the time derivatives in this operator.

Let $V^{k}(s, y)$ denote a function approximating $V_{M}\left(s, y, t_{k}\right), k=0,1, \ldots, M-1$. Note that the subscript $M$ has been removed from $V^{k}(s, y)$ to keep the notation simple. According to (3.3), we set $V^{M}(s, y)=\varsigma(s)$. Let us consider the following implicit-explicit (IMEX) time stepping scheme:

$$
\begin{aligned}
\mathcal{L} V^{k}(s, y)= & -\mathbf{F} \nabla V^{k}(s, y)+\nabla \cdot\left(\mathbf{E} \nabla V^{k}(s, y)\right)-(r+\lambda) V^{k}(s, y) \\
& +\lambda \int_{\mathbb{R}^{+}} V^{k+1}(s x, y) f(x) d x .
\end{aligned}
$$

For a detailed description of such an approach the reader is referred to [21]. Also we use

$$
\frac{\partial}{\partial t} V(s, y, t) \simeq \frac{V^{k+1}(s, y)-V^{k}(s, y)}{\Delta t}+\mathcal{O}(\Delta t)
$$


using relations (3.4) and (3.5), we define the following operator

$$
\begin{aligned}
& \tilde{\mathcal{L}} V^{k}(s, y) \simeq \frac{V^{k+1}(s, y)-V^{k}(s, y)}{\Delta t}+\mathcal{L} V^{k}(s, y) \\
& =\frac{1}{\Delta t} V^{k+1}(s, y)-\mathbf{F} \nabla V^{k}(s, y)+\nabla \cdot\left(\mathbf{E} \nabla V^{k}(s, y)\right) \\
& \quad-\left(r+\lambda+\frac{1}{\Delta t}\right) V^{k}(s, y)+\lambda \int_{\mathbb{R}^{+}} V^{k+1}(s x, y) f(x) d x,
\end{aligned}
$$

Therefore, the American option problems are rewritten as follows:

$$
\left\{\begin{array}{l}
\widetilde{\mathcal{L}} V^{k}(s, y)=0, \\
V^{k}(0, y)=0, \quad \lim _{s \rightarrow+\infty} V^{k}(s, y)=s-E
\end{array}\right.
$$

and also, the relations (3.2) and (3.3) are rewritten as follows:

$$
\begin{aligned}
& V^{k}(s, y)=\max \left(\lim _{t \rightarrow t_{k+1}^{+}} V^{k}(s, y), \varsigma(s)\right), \quad k=M-1, M-2, \ldots, 1,0 \\
& V^{M}(s, y)=\varsigma(s) .
\end{aligned}
$$

Remark 1: Note that in relation (3.4), the integral part is a non-local integral, whereas the other parts which are differential operators, are all local. No doubt, since the integral part is non-local operator, a dense linear system of equations will be obtained by using the $\theta$-weighted discretization scheme. Therefore, to obtain a sparse linear system of equations, it is better to use a IMEX scheme which is noted for avoiding dense matrices. So far published work existing in the literature which use the IMEX scheme to price the options, include [57]. Therefore, in this work, we use the IMEX scheme which is only first-order accurate in time. Then, the obtained approximation, which is only first-order accurate, is improved by Richardson extrapolation. In particular, we manage to obtain second-order accuracy by extrapolation of two solutions computed using $M$ and $2 M$ time steps. In the following, for the sake of brevity, we will restrict our attention to first stage of the Richardson extrapolation procedure, where $M$ time steps are employed, and the fact that the partial integro-differential problems considered are also solved with $2 M$ time steps will be understood.

\subsection{Spatial variable transformation}

It is well-known that from the mathematical point of view, the Bates stochastic volatility model typically leads to a partial integro-differential equation that is defined in the unbounded spatial domain $\mathbb{R}^{+} \times \mathbb{R}^{+}$. But due to the fact that unbounded domain cannot be discretized with finite amount of memory if the support of basis is compact, we replace the domain with the finite domain $\Omega=\left[0, S_{\max }\right] \times\left[0, y_{\max }\right]$ of the asset price and the volatility, where $S_{\max }$ and $y_{\max }$ are chosen sufficiently large to avoid an unacceptably

large truncation error. However, in [62] shown that upper bound of the asset price is three or four times of the strike price, so we can set $S_{\max }=4 E$. The options' payoffs considered in this paper are non-smooth functions, in particular their derivatives are discontinuous at the strike price. Therefore, to reduce the losses of accuracy the points of the trial functions are concentrated in a spatial region close to $s=E$. In contrast, along the $y$-direction, we want to have a mesh which is finer in a neighborhood of $y=y_{0}$, where the possible realizations of the variance process are more likely to occur [11]. In this work $y_{0}$ is the same value as is presented in [11] i.e. $y_{0}=0.04$. So, we employ the following change of variables:

$$
\begin{aligned}
& x(s)=\frac{\sinh ^{-1}\left(\xi_{s}(s-E)\right)+\sinh ^{-1}\left(\xi_{s} E\right)}{\sinh ^{-1}\left(\xi_{s}\left(S_{\text {max }}-E\right)\right)+\sinh ^{-1}\left(\xi_{s} E\right)}, \\
& z(y)=\frac{\sinh ^{-1}\left(\xi_{y}\left(y-y_{0}\right)\right)+\sinh ^{-1}\left(\xi_{y} y_{0}\right)}{\sinh ^{-1}\left(\xi_{y}\left(y_{\max }-y_{0}\right)\right)+\sinh ^{-1}\left(\xi_{y} y_{0}\right)},
\end{aligned}
$$



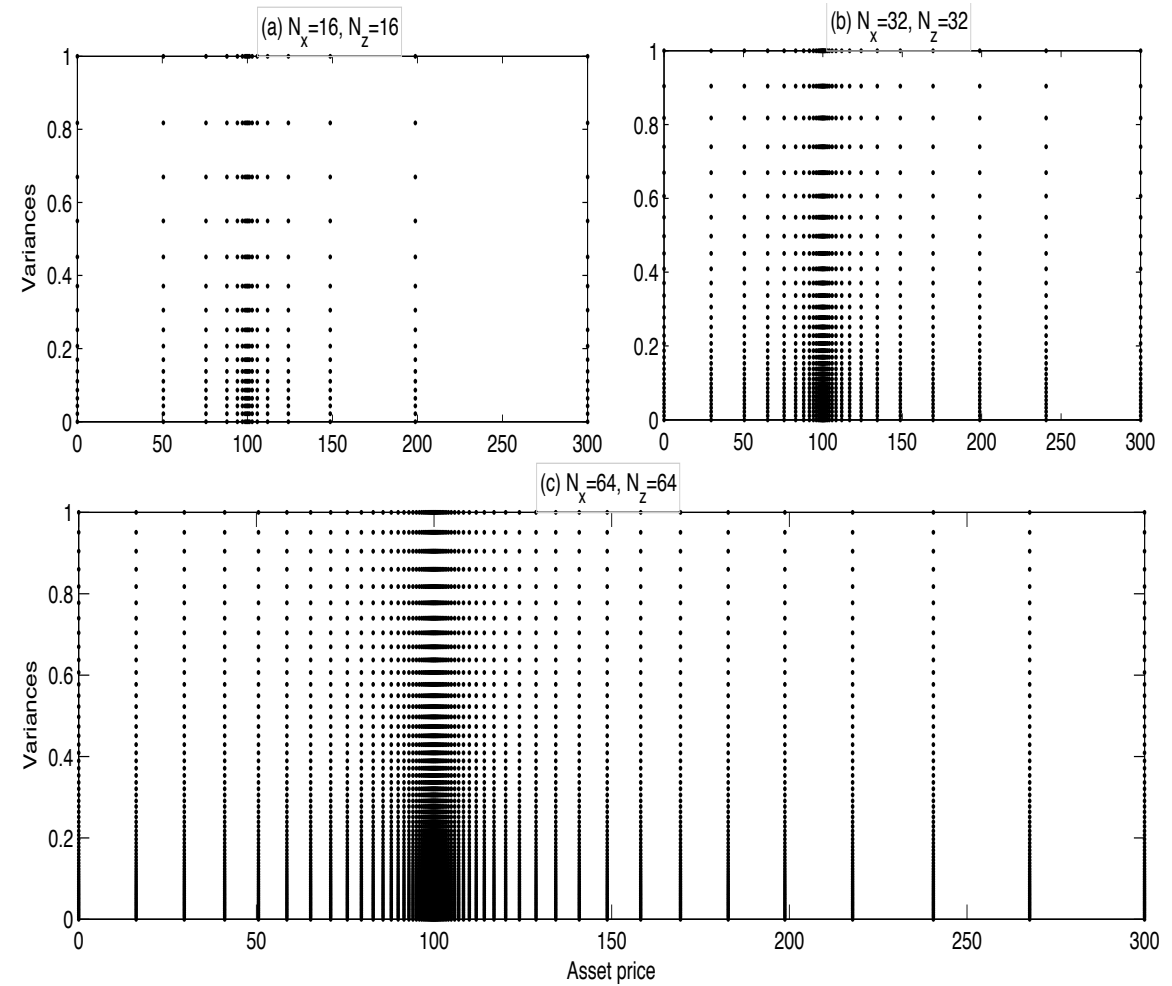

Figure 1: The distribution of nodes using $\xi_{s}=1, \xi_{y}=10, E=100, y_{0}=0.04, S_{\max }=3 E$ and $y_{\max }=1$.

or

$$
\begin{aligned}
& s(x)=\frac{1}{\xi_{s}} \sinh \left(x \sinh ^{-1}\left(\xi_{s}\left(S_{\max }-E\right)\right)-(1-x) \sinh ^{-1}\left(\xi_{s} E\right)\right)+E \\
& y(z)=\frac{1}{\xi_{y}} \sinh \left(z \sinh ^{-1}\left(\xi_{y}\left(y_{\max }-y_{0}\right)\right)-(1-z) \sinh ^{-1}\left(\xi_{y} y_{0}\right)\right)+y_{0},
\end{aligned}
$$

where $\xi_{s}$ and $\xi_{y}$ are the suitable constant parameters. Using these parameters, we can control the amount of the distribution of nodes in the $s$ and $y$-directions near $s=E$ and $y=y_{0}$, see Figure 1 .

Note that the relations $(3.9)$ and $(3.10)$ maps the $\left[0, S_{\max }\right] \times\left[0, y_{\max }\right]$ to the $[0,1] \times[0,1]$. We define

$$
\begin{aligned}
U(x, z, t)= & V(s(x), y(z), t), \\
\widetilde{\mathcal{L}} U^{k}(x, z)= & \frac{1}{\Delta t} U^{k+1}(x, z)-(\widetilde{\mathbf{F}} * \mathbf{P}) \nabla U^{k}(x, z) \\
& +\mathbf{P}\left[\nabla *\left(\left[\widetilde{\mathbf{E}} * \mathbf{P}^{T}\right]^{T} \nabla U^{k}(x, z)\right)\right] \\
& -\left(r+\lambda+\frac{1}{\Delta t}\right) U^{k}(x, z) \\
& +\lambda \int_{0}^{1} U^{k+1}(\widehat{r}, z) f\left(\frac{r(\widehat{r})}{s(x)}\right) \frac{1}{s(x)} r^{\prime}(\widehat{r}) d \widehat{r} \\
& +\lambda \int_{S_{\max }}^{\infty}(r-E) f\left(\frac{r}{s(x)}\right) \frac{1}{s(x)} d r,
\end{aligned}
$$




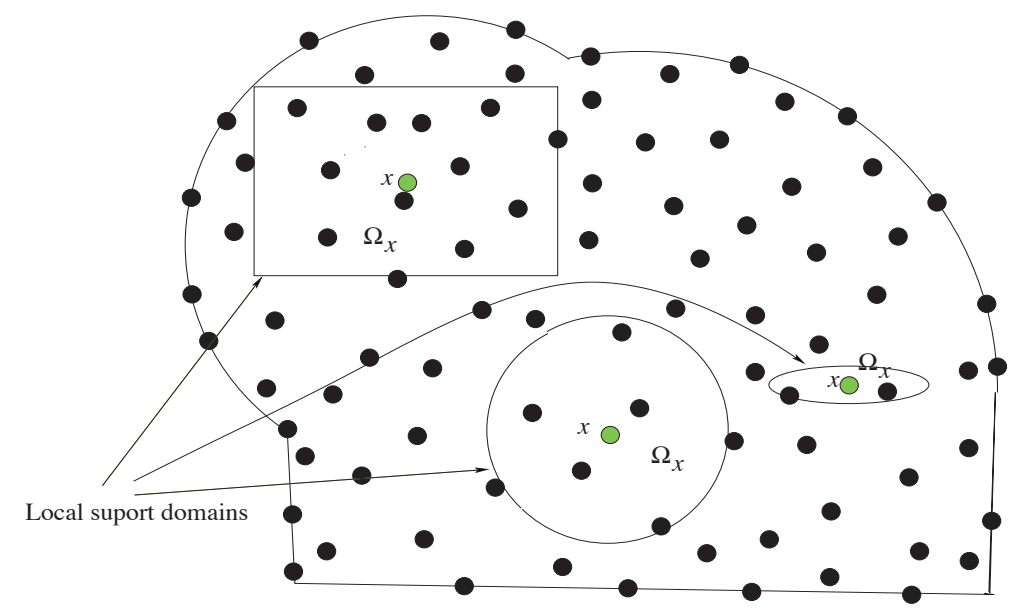

Figure 2: Support sub-domains of different points in the problem domain.

where the symbol $*$ means component-wise multiplication and also

$$
\begin{aligned}
& \widetilde{\mathbf{E}}=\frac{1}{2}\left(\begin{array}{cc}
y(z) s^{2}(x) & \rho \theta s(x) y(z) \\
\rho \theta s(x) y(z) & \theta^{2} y(z)
\end{array}\right), \\
& \widetilde{\mathbf{F}}=-\left(\begin{array}{c}
(r-q-\lambda \kappa) s(x)-y(z) s(x)-\rho \frac{\theta}{2} s(x) \\
\xi(\eta-y(z))-\frac{\theta^{2}}{2}-\rho \frac{\theta}{2} y(z)
\end{array}\right)^{T}, \\
& \mathbf{P}=\left(\begin{array}{c}
\frac{1}{s^{\prime}(x)} \\
\frac{1}{y^{\prime}(z)}
\end{array}\right)^{T}, \\
& r(\widehat{r})=\frac{1}{\xi_{s}} \sinh \left(\widehat{r} \sinh ^{-1}\left(\xi_{s}\left(S_{\text {max }}-E\right)\right)-(1-\widehat{r}) \sinh ^{-1}\left(\xi_{s} E\right)\right)+E,
\end{aligned}
$$

Using the change of variable (3.9), the relations (3.7) are rewritten as follows:

$$
\left\{\begin{array}{l}
\widetilde{\mathcal{L}} U^{k}(x, z)=0, \\
U^{k}(0, z)=0, \quad U^{k}(1, z)=S_{\max }-E .
\end{array}\right.
$$

and also, the relations (3.8) are rewritten as follows:

$$
\begin{aligned}
& U^{k}(x, z)=\max \left(\lim _{t \rightarrow t_{k+1}^{+}} U^{k}(x, z), \widetilde{\varsigma}(x)\right), \quad k=0,1, \ldots, M-1, \\
& U^{M}(x, z)=\widetilde{\varsigma}(x),
\end{aligned}
$$

where

$$
\widetilde{\varsigma}(x)=\max (s(x)-E, 0) .
$$

\subsection{The local weak form}

In this section, we use the local weak form instead of the global weak form. The local weak form meshless method was firstly proposed by Atluri et al. [6], in their meshless local Petrov-Galerkin method. The MLPG method constructs the weak form over local sub-domains such as $\Omega_{s}$, which is a small region taken for each node in the global domain $\Omega=[0,1] \times[0,1]$. The local sub-domains overlap each other and cover the whole global domain $\Omega$. This local sub-domains could be any simple geometry like a circle, square, as shown in Figure 2. For simplicity, we assume the local sub-domains have circular shape. Therefore the local weak form of the approximate equation (3.15) for $\mathbf{x} \in \Omega_{s}^{i}$, where $\mathbf{x}=(x, z)$, can be written as

$$
<\widetilde{\mathcal{L}} U^{k}, u^{\star}>=0,
$$


In equation $(3.18), u^{\star}$ is the indicator function

$$
u^{\star}(\mathbf{x})= \begin{cases}1 & \mathbf{x} \in \Omega_{s}^{i}, \\ 0 & \text { o.w. }\end{cases}
$$

as the test function in each local domain. Also we define the inner product $<., .>$ on interior domain and $\{.,$.$\} on boundary as$

$$
\begin{aligned}
& <\widetilde{\mathcal{L}} U^{k}, u^{\star}>=\int_{\Omega_{s}^{i}} \widetilde{\mathcal{L}} U^{k}(\mathbf{x}) u^{\star}(\mathbf{x}) d \Omega, \\
& \left\{\widetilde{\mathcal{L}} U^{k}, u^{\star}\right\}=\int_{\partial \Omega_{s}^{i}} \widetilde{\mathcal{L}} U^{k}(\mathbf{x}) u^{\star}(\mathbf{x}) d \Gamma,
\end{aligned}
$$

which $\Omega_{s}^{i}$ is the local domain associated with the point $i$, i.e. it is a circle centered at $\mathbf{x}$ of radius $r_{Q}$. Let $\partial \Omega_{s}^{i}$ denote the boundary of sub-domain $\Omega_{s}^{i}$.

Eq. (3.19) with the substitution relation (3.13) constitute the following relation

$$
\begin{aligned}
& <(\widetilde{\mathbf{F}} * \mathbf{P}) \nabla U^{k}, u^{\star}>-<\mathbf{P}\left[\nabla *\left(\left[\widetilde{\mathbf{E}} * \mathbf{P}^{T}\right]^{T} \nabla U^{k}(x, z)\right)\right], u^{\star}> \\
& +\left(r+\lambda+\frac{1}{\Delta t}\right)<U^{k}, u^{\star}> \\
& =\frac{1}{\Delta t}<U^{k+1}, u^{\star}>+\lambda<\mathcal{W}, u^{\star}>+\lambda<\Pi, u^{\star}>
\end{aligned}
$$

where

$$
\begin{aligned}
& \mathcal{W}(z)=\int_{0}^{1} U^{k+1}(\widehat{r}, z) f\left(\frac{r(\widehat{r})}{s(x)}\right) \frac{1}{s(x)} r^{\prime}(\widehat{r}) d \widehat{r}, \\
& \Pi=\int_{S_{\max }}^{\infty}(r-E) f\left(\frac{r}{s(x)}\right) \frac{1}{s(x)} d r .
\end{aligned}
$$

An alternative for $<\mathbf{P}\left[\nabla *\left(\left[\widetilde{\mathbf{E}} * \mathbf{P}^{T}\right]^{T} \nabla U^{k}(x, z)\right)\right], u^{\star}>$ related to relation (3.20) can be mentioned as

$$
\begin{aligned}
& <\mathbf{P}\left[\nabla *\left(\left[\widetilde{\mathbf{E}} * \mathbf{P}^{T}\right]^{T} \nabla U^{k}(x, z)\right)\right], u^{\star}>= \\
& <\left[\mathbf{P} *\left[\nabla *\left(\mathbf{P} \widetilde{\mathbf{E}}_{1}\right)+\widetilde{\mathbf{E}}_{2}\right]\right] \nabla U^{k}, u^{\star}> \\
& +<[\operatorname{Diag}(\widetilde{\mathbf{E}}) * \mathbf{P} * \mathbf{P}] \Delta \cdot U^{k}, u^{\star}>+\rho \theta<\frac{s(x) y(z)}{s^{\prime}(x) y^{\prime}(z)} \frac{\partial^{2}}{\partial x \partial z} U^{k}, u^{\star}>,
\end{aligned}
$$

where

$$
\begin{aligned}
& \widetilde{\mathbf{E}}_{1}=\frac{1}{2}\left(\begin{array}{cc}
y(z) s^{2}(x) & 0 \\
0 & \theta^{2} y(z)
\end{array}\right), \\
& \widetilde{\mathbf{E}}_{2}=\frac{1}{2} \rho \theta\left(\begin{array}{c}
s(x) \\
y(z)
\end{array}\right)^{T}, \\
& \Delta \cdot U^{k}=\left(\begin{array}{c}
\frac{\partial^{2}}{\partial x^{2}} U^{k} \\
\frac{\partial^{2}}{\partial z^{2}} U^{k}
\end{array}\right)^{T} .
\end{aligned}
$$


What will be used in here for simplifying the system (3.20) is the divergence theorem as follows

$$
\begin{aligned}
& <(\widetilde{\mathbf{F}} * \mathbf{P}) \nabla U^{k}, u^{\star}>=-<[\nabla \cdot(\widetilde{\mathbf{F}} * \mathbf{P})] U^{k}, u^{\star}>+\left\{[(\widetilde{\mathbf{F}} * \mathbf{P}) \cdot \nu] U^{k}, u^{\star}\right\} \\
& <\left[\mathbf{P} *\left[\nabla *\left(\mathbf{P} \widetilde{\mathbf{E}}_{1}\right)+\widetilde{\mathbf{E}}_{2}\right]\right] \nabla U^{k}, u^{\star}>= \\
& \quad-\quad<\nabla \cdot\left[\mathbf{P} *\left[\nabla *\left(\mathbf{P} \widetilde{\mathbf{E}}_{1}\right)+\widetilde{\mathbf{E}}_{2}\right]\right] U^{k}, u^{\star}> \\
& \quad+\left\{\left[\mathbf{P} *\left[\nabla *\left(\mathbf{P} \widetilde{\mathbf{E}}_{1}\right)+\widetilde{\mathbf{E}}_{2}\right]\right] \cdot \nu U^{k}, u^{\star}\right\}, \\
& <[\operatorname{Diag}(\widetilde{\mathbf{E}}) * \mathbf{P} * \mathbf{P}] \Delta \cdot U^{k}, u^{\star}>=<\Delta \cdot(\operatorname{Diag}(\widetilde{\mathbf{E}}) * \mathbf{P} * \mathbf{P}) U^{k}, u^{\star}> \\
& -\left\{[[\nabla *[\operatorname{Diag}(\widetilde{\mathbf{E}}) * \mathbf{P} * \mathbf{P}]] \cdot \nu] U^{k}, u^{\star}\right\} \\
& \quad+\left\{\left[[\operatorname{Diag}(\widetilde{\mathbf{E}}) * \mathbf{P} * \mathbf{P}] * \nabla U^{k}\right] \cdot \nu, u^{\star}\right\}, \\
& <\frac{s(x) y(z)}{s^{\prime}(x) y^{\prime}(z)} \frac{\partial^{2}}{\partial x \partial z} U^{k}, u^{\star}>=<\left(\frac{s(x)}{s^{\prime}(x)}\right)^{\prime}\left(\frac{y(z)}{y^{\prime}(z)}\right)^{\prime} U^{k}, u^{\star}> \\
& \quad-\left\{\left(\frac{s(x)}{s^{\prime}(x)}\right)\left(\frac{y(z)}{y^{\prime}(z)}\right)^{\prime} U^{k} \nu^{1}, u^{\star}\right\} \\
& +\left\{\frac{s(x) y(z)}{s^{\prime}(x) y^{\prime}(z)} \frac{\partial}{\partial x} U^{k} \nu^{2}, u^{\star}\right\}
\end{aligned}
$$

where $\nu$ is unit outward normal vector on the boundary of the domain of the problem. Substituting relations (3.22) in (3.20), we obtain

$$
\begin{aligned}
& -<[\nabla \cdot(\widetilde{\mathbf{F}} * \mathbf{P})] U^{k}, u^{\star}>+<\nabla \cdot\left[\mathbf{P} *\left[\nabla *\left(\mathbf{P} \widetilde{\mathbf{E}}_{1}\right)+\widetilde{\mathbf{E}}_{2}\right]\right] U^{k}, u^{\star}> \\
& -<\Delta \cdot(\operatorname{Diag}(\widetilde{\mathbf{E}}) * \mathbf{P} * \mathbf{P}) U^{k}, u^{\star}>-<\left(\frac{s(x)}{s^{\prime}(x)}\right)^{\prime}\left(\frac{y(z)}{y^{\prime}(z)}\right)^{\prime} U^{k}, u^{\star}> \\
& +\left(r+\lambda+\frac{1}{\Delta t}\right)<U^{k}, u^{\star}>+\left\{[(\widetilde{\mathbf{F}} * \mathbf{P}) \cdot \nu] U^{k}, u^{\star}\right\} \\
& -\left\{\left[\mathbf{P} *\left[\nabla *\left(\mathbf{P} \widetilde{\mathbf{E}}_{1}\right)+\widetilde{\mathbf{E}}_{2}\right]\right] . \nu U^{k}, u^{\star}\right\} \\
& +\left\{[[\nabla *[\operatorname{Diag}(\widetilde{\mathbf{E}}) * \mathbf{P} * \mathbf{P}]] . \nu] U^{k}, u^{\star}\right\}-\left\{\left[[\operatorname{Diag}(\widetilde{\mathbf{E}}) * \mathbf{P} * \mathbf{P}] * \nabla U^{k}\right] . \nu, u^{\star}\right\} \\
& +\left\{\left(\frac{s(x)}{s^{\prime}(x)}\right)\left(\frac{y(z)}{y^{\prime}(z)}\right)^{\prime} U^{k} \nu^{1}, u^{\star}\right\}-\left\{\frac{s(x) y(z)}{s^{\prime}(x) y^{\prime}(z)} \frac{\partial}{\partial x} U^{k} \nu^{2}, u^{\star}\right\} \\
& =\frac{1}{\Delta t}<U^{k+1}, u^{\star}>+\lambda<\mathcal{W}, u^{\star}>+\lambda<\Pi, u^{\star}>
\end{aligned}
$$

It is important to observe that in relation (3.23) exist unknown functions, we should approximate these functions. To this aim the local integral equations (3.23) are transformed in to a system of algebraic equations with real unknown quantities at nodes used for spatial approximation, as described in the next subsection.

\subsection{Spatial approximation}

Rather than using traditional non-overlapping, contiguous meshes to make the interpolation scheme, the MLPG method uses a local interpolation or approximation to represent the trial or test functions with the values (or the fictitious values) of the unknown variable at some randomly located nodes. We will find a number of local interpolation schemes for this purpose. The radial point interpolation method is certainly one of them. The LRPI scheme is utilized in this paper. In this section, the fundamental idea of the LRPI is reviewed.

Consider a subdomain $\Omega_{\mathbf{x}}$ of $\Omega=[0,1] \times[0,1]$ in the neighborhood of a point $\mathbf{x}$ for the definition of the LRPI approximation of the trial function around $\mathbf{x}$.

According to the local point interpolation [42], the value of point interpolation approximation of $U^{k}(\mathbf{x})$ at any (given) point $\mathbf{x} \in \Omega$ is approximated by interpolation at $n$ nodes $\mathbf{x}_{1}, \mathbf{x}_{2}, \ldots, \mathbf{x}_{n}$ (centers) laying in a convenient neighborhood of $\mathbf{x}$ i.e. $\Omega_{\mathbf{x}}$. The domain in which these nodes are chosen, whose shape may depend on the point $\mathbf{x}$, is usually referred to as local support domain. Various different local point interpolation approaches can be obtained depending on the functions used to interpolate $U^{k}(\mathbf{x})$. In this 
paper we focus our attention onto the so-called local radial point interpolation method (LRPI), which employs a combination of polynomials and radial basis functions.

To approximate the distribution of function $U^{k}(\mathbf{x})$ in $\Omega_{\mathbf{x}}$, over a number of randomly located nodes $\left\{\mathbf{x}_{i}\right\}, \quad i=1,2, \ldots, n$, the radial point interpolation approximation $\widetilde{U}^{k}(\mathbf{x})$ of $U^{k}(\mathbf{x})$ for each $\mathbf{x} \in \Omega_{\mathbf{x}}$, can be defined by

$$
\widetilde{U}^{k}(\mathbf{x})=\sum_{i=1}^{n} R_{i}(\mathbf{x}) a_{i}^{k}+\sum_{j=1}^{m} P_{j}(\mathbf{x}) b_{j}^{k},
$$

where $P_{1}, P_{2}, \ldots, P_{m}$ denote the first $m$ monomials in ascending order and $R_{1}, R_{2}, \ldots, R_{n}$ are $n$ radial functions centered at $\mathbf{x}_{1}, \mathbf{x}_{2}, \ldots, \mathbf{x}_{n}$, respectively. Moreover $a_{1}^{k}, a_{2}^{k}, \ldots, a_{n}^{k}, b_{1}^{k}, b_{2}^{k}, \ldots, b_{m}^{k}$ are $n+m$ real coefficients that have to be determined.

As far as the radial basis functions $R_{1}, R_{1}, \ldots, R_{n}$ are concerned, several choices are possible (see, for example, [14]). In this work we decide to use the Wendland's compactly supported radial basis functions (WCS-RBFs) with $C^{6}, C^{4}$ and $C^{2}$ smoothness [61], as they do not involve any free shape parameter (which is not straightforward to choose, see $[16,15,27,9,8]$ ). WCS-RBFs with $C^{6}, C^{4}$ and $C^{2}$ smoothness degrees are as follows, respectively:

$$
\begin{aligned}
& R_{i}(s)=\left(1-r_{i}\right)_{+}^{4}\left(1+4 r_{i}\right), \quad i=1,2, \ldots, n, \\
& R_{i}(s)=\left(1-r_{i}\right)_{+}^{6}\left(3+18 r_{i}+35 r_{i}^{2}\right), \quad i=1,2, \ldots, n, \\
& R_{i}(s)=\left(1-r_{i}\right)_{+}^{8}\left(1+8 r_{i}+25 r_{i}^{2}+32 r_{i}^{3}\right), \quad i=1,2, \ldots, n,
\end{aligned}
$$

where $r_{i}=\left\|\mathbf{x}-\mathbf{x}_{i}\right\| / r_{w}^{i}$ is the distance from node $\mathbf{x}_{i}$ to $\mathbf{x}$, while $r_{w}^{i}$ is the size of support for the radial function $R_{i}(\mathbf{x})$. In this study, for simplicity, we set $r_{w}^{i}=r_{w}$ for all $i$. Also, $\left(1-r_{i}\right)_{+}^{l}$ is $\left(1-r_{i}\right)^{l}$ for $0 \leq r_{i}<1$ and zero otherwise.

Note that the monomials $P_{1}, P_{2}, \ldots, P_{m}$ are not always employed (if $b_{i}^{k}=0, i=1,2, \ldots, m$, pure RBF approximation is obtained). In the present work, both the constant and the linear monomials are used to augment the RBFs (i.e. we set $m=4$ ). Note that, with respect to the RBF meshless methods employed in $[36,10,32,29,63,43]$, the radial basis point interpolation which is a basis of both polynomials and radial functions offers the following advantages: First of all, it incorporates polynomial terms in the basis, which are useful to reduce the ill-conditioning of the resulting linear systems. Second, it possesses the Kronecker property, so that essential boundary and initial (or final) conditions can be easily imposed (it should be noted that the Kronecker delta is a two variables function $\delta(x, y)$ such that its value is 1 if $x=y$ and 0 otherwise). Third, it can be reinterpreted as a Kriging method [38], and thus its interpolation error can be considered optimal also from a statistical point of view.

By requiring that the function $\widetilde{U}^{k}$ interpolate $U$ at $\mathbf{x}_{1}, \mathbf{x}_{2}, \ldots, \mathbf{x}_{n}$, we obtain a set of $n$ equations in the $n+m$ unknown coefficients $a_{1}^{k}, a_{2}^{k}, \ldots, a_{n}^{k}, b_{1}^{k}, b_{2}^{k}, \ldots, b_{m}^{k}$ :

$$
\sum_{i=1}^{n} R_{i}\left(\mathbf{x}_{p}\right) a_{i}^{k}+\sum_{j=1}^{m} P_{j}\left(\mathbf{x}_{p}\right) b_{j}^{k}=\widehat{U}^{k}\left(\mathbf{x}_{p}\right), \quad p=1,2, \ldots, n
$$

where $\widehat{U}^{k}$ are the fictitious nodal nodes.

Moreover, in order to uniquely determine $\widetilde{U}^{k}$, we also impose:

$$
\sum_{i=1}^{n} P_{j}\left(\mathbf{x}_{i}\right) a_{i}^{k}=0, \quad j=1,2, \ldots, m .
$$

That is we have the following system of linear equations:

$$
\mathbf{G}\left[\begin{array}{l}
\mathbf{a}^{k} \\
\mathbf{b}^{k}
\end{array}\right]=\left[\begin{array}{c}
\widehat{\mathbf{U}}^{k} \\
\mathbf{0}
\end{array}\right],
$$


where

$$
\begin{aligned}
& \widehat{\mathbf{U}}^{k}=\left[\begin{array}{llll}
\widehat{U}_{1}^{k} & \widehat{U}_{2}^{k} & \ldots & \widehat{U}_{n}^{k}
\end{array}\right]^{T}=\left[\begin{array}{llll}
\widehat{U}^{k}\left(\mathbf{x}_{1}\right) & \widehat{U}^{k}\left(\mathbf{x}_{2}\right) & \ldots & \widehat{U}^{k}\left(\mathbf{x}_{n}\right)
\end{array}\right]^{T} \\
& \mathbf{G}=\left[\begin{array}{cc}
\mathbf{R} & \mathbf{P} \\
\mathbf{P}^{T} & \mathbf{0}
\end{array}\right] \\
& \mathbf{R}=\left[\begin{array}{cccc}
R_{1}\left(\mathbf{x}_{1}\right) & R_{2}\left(\mathbf{x}_{1}\right) & \ldots & R_{n}\left(\mathbf{x}_{1}\right) \\
R_{1}\left(\mathbf{x}_{2}\right) & R_{2}\left(\mathbf{x}_{2}\right) & \ldots & R_{n}\left(\mathbf{x}_{2}\right) \\
\vdots & \vdots & \ddots & \vdots \\
R_{1}\left(\mathbf{x}_{n}\right) & R_{2}\left(\mathbf{x}_{n}\right) & \ldots & R_{n}\left(\mathbf{x}_{n}\right)
\end{array}\right] \\
& \mathbf{P}=\left[\begin{array}{cccc}
P_{1}\left(\mathbf{x}_{1}\right) & P_{2}\left(\mathbf{x}_{1}\right) & \ldots & P_{m}\left(\mathbf{x}_{1}\right) \\
P_{1}\left(\mathbf{x}_{2}\right) & P_{2}\left(\mathbf{x}_{2}\right) & \ldots & P_{m}\left(\mathbf{x}_{2}\right) \\
\vdots & \vdots & \ddots & \vdots \\
P_{1}\left(\mathbf{x}_{n}\right) & P_{2}\left(\mathbf{x}_{n}\right) & \ldots & P_{m}\left(\mathbf{x}_{n}\right)
\end{array}\right] \\
& \mathbf{a}^{k}=\left[\begin{array}{llll}
a_{1}^{k} & a_{2}^{k} & \ldots & a_{n}^{k}
\end{array}\right]^{T} \\
& \mathbf{b}^{k}=\left[\begin{array}{llll}
b_{1}^{k} & b_{2}^{k} & \ldots & b_{m}^{k}
\end{array}\right]^{T},
\end{aligned}
$$

Unique solution is obtained if the inverse of matrix $\mathbf{R}$ exists [61], so that

$$
\left[\begin{array}{l}
\mathbf{a}^{k} \\
\mathbf{b}^{k}
\end{array}\right]=\mathbf{G}^{-1}\left[\begin{array}{c}
\widehat{\mathbf{U}}^{k} \\
\mathbf{0}
\end{array}\right] .
$$

Accordingly, (3.24) can be rewritten as

$$
\widetilde{U}^{k}(x)=\left[\begin{array}{ll}
\mathbf{R}^{T}(\mathbf{x}) & \mathbf{P}^{T}(\mathbf{x})
\end{array}\right]\left[\begin{array}{l}
\mathbf{a}^{k} \\
\mathbf{b}^{k}
\end{array}\right],
$$

or, equivalently,

$$
\widetilde{U}^{k}(\mathbf{x})=\left[\begin{array}{ll}
\mathbf{R}^{T}(\mathbf{x}) & \mathbf{P}^{T}(\mathbf{x})
\end{array}\right] \mathbf{G}^{-1}\left[\begin{array}{c}
\widehat{\mathbf{U}}^{k} \\
\mathbf{0}
\end{array}\right] .
$$

Let us define the vector of shape functions:

$$
\boldsymbol{\Phi}(\mathbf{x})=\left[\begin{array}{llll}
\varphi_{1}(\mathbf{x}) & \varphi_{2}(\mathbf{x}) & \ldots & \varphi_{n}(\mathbf{x})
\end{array}\right],
$$

where

$$
\varphi_{p}(\mathbf{x})=\sum_{i=1}^{n} R_{i}(\mathbf{x}) \mathbf{G}_{i, p}^{-1}+\sum_{j=1}^{m} P_{j}(\mathbf{x}) \mathbf{G}_{n+j, p}^{-1}, \quad p=1,2, \ldots, n
$$


and $\mathbf{G}_{i, p}^{-1}$ is the $(i, p)$ element of the matrix $\mathbf{G}^{-1}$.

Using (3.31) relations (3.30) are rewritten in the more compact form:

$$
\widetilde{U}^{k}(\mathbf{x})=\mathbf{\Phi}(\mathbf{x}) \widehat{\mathbf{U}}^{k}
$$

or, equivalently,

$$
\widetilde{U}^{k}(\mathbf{x})=\sum_{i=1}^{n} \widehat{U}_{i}^{k} \varphi_{i}(\mathbf{x})
$$

It can be easily shown that the shape functions (3.31) satisfy the so-called Kronecker property, that is

$$
\varphi_{i}\left(\mathbf{x}_{j}\right)=\delta_{i j}
$$

where $\delta_{i j}$ is the well-known Kronecker symbol, so that essential boundary and final conditions such as those considered in Section 2 (e.g., relation (3.15)) can be easily imposed. Note also that the derivatives of $\widetilde{U}^{k}$ (of any order) with respect to $x$ or $z$ are easily obtained by direct differentiation in (3.33).

\subsection{Discretized equations}

Before we show how to discretize model in the form (3.23), we focus on how to select nodal points. Let $X=\left\{\mathbf{x}_{0}, \mathbf{x}_{1}, \ldots, \mathbf{x}_{N}\right\} \subset \Omega$ are scattered meshless points, where some points are located on the boundary to enforce the boundary conditions. In fact, $\mathbf{x}_{0}, \mathbf{x}_{N} \in \partial \Omega$. The options' payoffs considered in this paper are non-smooth functions, in particular their derivatives are discontinuous at the strike price. Therefore, to reduce the losses of accuracy due to such a non-smooth initial datum, the points of the trial functions are concentrated in a spatial region close to $s=E$. So, this is satisfied using relation (3.11) and the following uniform nodal points along the $x$ and the $z$ directions, respectively:

$$
\begin{aligned}
& x_{i}=i \Delta x, \quad i=0,1, \ldots, N_{x}, \\
& z_{j}=j \Delta z, \quad j=0,1, \ldots, N_{z},
\end{aligned}
$$

where $\Delta x=1 / N_{x}, \Delta z=1 / N_{z}$ and $N=\left(N_{x}+1\right)\left(N_{z}+1\right)$. It is important to observe that $U^{k+1}(\mathbf{x})$ must be considered as known quantities, since it is approximated at the previous iteration. We want to approximate $U^{k}(\mathbf{x})$ using LRPI approximation. In the MLPG scheme, it is easy to enforce the boundary conditions (3.7) for that the shape function constructed by the LRPI approximation. The LRPI approximation has shape functions with delta function properties, thus it allows one to easily impose essential boundary and initial (or final) conditions.

Substituting the displacement expression in Eq. (3.32) into the local weak form (3.23) for each interior node in $\Omega_{s}^{i}$ the matrix forms of the their discrete equations are obtained as follows

$$
\mathbf{F} \widehat{\mathbf{U}}^{k}=\mathbf{G} \widehat{\mathbf{U}}^{k+1}
$$

where

$$
\widehat{\mathbf{U}}^{k}=\left[\begin{array}{lllll}
\widehat{U}_{N_{z}+1}^{k} & \widehat{U}_{N_{z}+2}^{k} & \widehat{U}_{2}^{k} & \ldots & \widehat{U}_{N-N_{z}-1}^{k}
\end{array}\right]_{\left(N-2 N_{z}-1\right) \times 1}^{T} .
$$

Again we should note that in relation (3.37), $\widehat{U}_{0}^{k}, \widehat{U}_{1}^{k}, \ldots, \widehat{U}_{N_{z}}^{k}$ and

$\widehat{U}_{N-N_{z}}^{k}, \widehat{U}_{N-N_{z}+1}^{k}, \ldots, \widehat{U}_{N}^{k}$ are calculated using the delta function properties easily. Also in the linear system (3.36), $\mathbf{G}=\left[\begin{array}{lllll}\mathbf{G}_{N_{z}+1} & \mathbf{G}_{N_{z}+2} & \ldots & \mathbf{G}_{N-N_{z}-1}\end{array}\right]^{T}$ is the $\left(N-2 N_{z}-1\right) \times\left(N-2 N_{z}-1\right)$ banded matrix with bandwith bw such that

$$
\mathbf{G}_{i}=\frac{1}{\Delta t} \widetilde{\mathbf{E}}_{i}+\sum_{l=0}^{N} \widetilde{\mathbf{L}}_{i}, \quad i=N_{z}+1, \ldots, N-N_{z}-1,
$$


where

$$
\left\{\widetilde{\mathbf{E}}_{i}\right\}_{j}= \begin{cases}\widetilde{\mathbf{E}}_{i j}, & \mathbf{x}_{j} \in X \cap \Omega_{s}^{i}, \\ 0, & \text { o.w. }\end{cases}
$$

This piecewise function which is defined to $\left\{\widetilde{\mathbf{E}}_{i}\right\}$, is extensible to ${ }_{l} \widetilde{\mathbf{L}}_{i}$. Also $\mathbf{F}=\left[\begin{array}{llll}\mathbf{F}_{N_{z}+1} & \mathbf{F}_{N_{z}+2} & \ldots & \mathbf{F}_{N-N_{z}-1}\end{array}\right]^{T}$ is the $\left(N-2 N_{z}-1\right) \times\left(N-2 N_{z}-1\right)$ banded matrix with bandwith bw. We have

$$
\mathbf{F}_{i}=\widetilde{\mathbf{A}}_{i}+\widetilde{\mathbf{B}}_{i}+\widetilde{\mathbf{C}}_{i}+\widetilde{\mathbf{D}}_{i}+\left(r+\lambda+\frac{1}{\Delta t}\right) \widetilde{\mathbf{E}}_{i}, \quad i=N_{z}+1, \ldots, N-N_{z}-1 .
$$

Again we do emphasize that the piecewise function which is defined to $\left\{\widetilde{\mathbf{E}}_{i}\right\}$ in relation (3.38), is extensible to $\widetilde{\mathbf{A}}_{i}, \widetilde{\mathbf{B}}_{i}, \widetilde{\mathbf{C}}_{i}$ and $\widetilde{\mathbf{D}}_{i}$. Also we can easily see that

$$
\begin{aligned}
\widetilde{\mathbf{A}}_{i j} & =\int_{\Omega_{s}^{i}} \mathbf{M}(\mathbf{x}) \varphi_{j}(\mathbf{x}) d \Omega, \quad \widetilde{\mathbf{B}}_{i j}=\int_{\partial \Omega_{s}^{i}} \mathbf{N}(\mathbf{x}) \varphi_{j}(\mathbf{x}) d \Gamma \\
\widetilde{\mathbf{C}}_{i j} & =\int_{\partial \Omega_{s}^{i}} \mathbf{I}(\mathbf{x}) \frac{\partial}{\partial x} \varphi_{j}(\mathbf{x}) d \Gamma, \quad \widetilde{\mathbf{D}}_{i j}=\int_{\partial \Omega_{s}^{i}} \boldsymbol{\Theta}(\mathbf{x}) \frac{\partial}{\partial z} \varphi_{j}(\mathbf{x}) d \Gamma, \\
{ }_{l} \widetilde{\mathbf{L}}_{i j} & =\lambda \int_{\Omega_{s}^{i}} \int_{\Omega_{s}^{l}} \frac{r^{\prime}(\hat{r})}{s(x)} f\left(\frac{\hat{r}}{s(x)}\right) \varphi_{j}(\hat{r}, z) d \hat{r} d \Omega, \\
\widetilde{\mathbf{E}}_{i j} & =\int_{\Omega_{s}^{i}} \varphi_{j}(\mathbf{x}) d \Omega
\end{aligned}
$$

where

$$
\begin{aligned}
& \mathbf{M}(\mathbf{x})=(r-q-\lambda \kappa)\left[\frac{s(x)}{s^{\prime}(x)}\right]^{\prime}+\xi\left[\frac{\eta-y(z)}{y^{\prime}(z)}\right]^{\prime}+\frac{1}{2} y(z)\left[\frac{s^{2}(x) s^{\prime \prime}(x)}{\left(s^{\prime}(x)\right)^{3}}\right] \\
& -y(z)\left[\frac{s(x)}{s^{\prime}(x)}\right]^{\prime}-\frac{\theta^{2}}{2} \frac{1}{y^{\prime}(z)}+\frac{1}{2} \theta^{2} \frac{y(z) y^{\prime \prime}(z)}{\left(y^{\prime}(z)\right)^{3}}-\left[\frac{s(x)}{s^{\prime}(x)}\right]\left[\frac{y(z)}{y^{\prime}(z)}\right]^{\prime}, \\
& \mathbf{N}(\mathbf{x})=-(r-q-\lambda \kappa) \frac{s(x)}{s^{\prime}(x)} \nu^{1}-\xi \frac{\eta-y(z)}{y^{\prime}(z)} \nu^{2}+\frac{s(x)}{s^{\prime}(x)}\left[\frac{y(z)}{y^{\prime}(z)}\right]^{\prime} \nu^{1} \\
& +\frac{1}{2} y(z) \frac{s^{2}(x)}{s^{\prime}(x)}\left[\frac{1}{s^{\prime}(x)}\right]^{\prime} \nu^{1}+y(z) \frac{s(x)}{s^{\prime}(x)} \nu^{1} \\
& +\frac{1}{2} \theta^{2} \frac{1}{y^{\prime}(z)} \nu^{2}+\frac{1}{2} \frac{y(z)}{y^{\prime}(z)}\left[\frac{1}{y^{\prime}(z)}\right]^{\prime} \nu^{2}, \\
& \mathbf{I}(\mathbf{x})=-\frac{1}{2} y(z)\left[\frac{s(x)}{s^{\prime}(x)}\right]^{2} \nu^{1}-\frac{s(x) y(z)}{s^{\prime}(x) y^{\prime}(z)} \nu^{2}, \\
& \mathbf{\Theta}(\mathbf{x})=-\frac{1}{2} \theta^{2} \frac{y(z)}{\left(y^{\prime}(z)\right)^{2}} \nu^{2},
\end{aligned}
$$

Finally, combining Eqs. (3.16) and (3.36) lead to the following system:

$$
\left\{\begin{array}{l}
\mathbf{F} \widehat{\boldsymbol{\Xi}}^{k}=\mathbf{G} \widehat{\mathbf{U}}^{k+1}, \\
\widehat{\mathbf{U}}^{k}=\max \left\{\widehat{\boldsymbol{\Xi}}^{k}, \widehat{\boldsymbol{\Pi}}\right\},
\end{array}\right.
$$

to be recursively solved for $k=M-1, M-2, \ldots, 0$, starting from

$$
\widehat{\mathbf{U}}^{M}=\widehat{\Pi},
$$


where $\widehat{\boldsymbol{\Pi}}$ are obtained from delta function properties of LRPI approximation and option's payoff (3.17).

Remark 2: The numerical method proposed in this work require solving at every time step a system of linear equations (systems (3.36)). Now, the matrix $\mathbf{F}$ associated to this system is band with bandwidth bw and well-conditioned, therefore, the aforementioned linear system is solved using the banded LU factorization method with partial pivoting, which is particularly suitable for banded matrices. It should also be noted that the complexity of banded LU factorization method with partial pivoting is $\mathcal{O}(2 N(2 \mathrm{bw}+3)(2 \mathrm{bw}+5))$. We simply observe that complexity of this algorithm is very lower than complexity of LU factorization method with partial pivoting for strong form of MLPG or global RBF method which is $\mathcal{O}\left(N^{3} / 3\right)$, see [30]. Moreover, as the matrix $\mathbf{F}$ to be inverted are the same for every time step, the banded LU factorization can be performed only once at the beginning of the numerical simulation, and thus at each time step the corresponding linear system is efficiently solved by forward and backward recursion (see [30]).

Remark 3: A crucial point in the MLPG is an accurate evaluation of the local integrals. Since the nodal trial functions based on LRPI are highly complicated, an accurate numerical integration of the weak form is highly difficult. In this work, the numerical integration procedure used is 4 points Gauss-Legendre quadrature rule by a suitable change of variables.

\section{Numerical results and discussions}

To get a better sense of the efficiency of the method presented in the current paper, let us employ the scheme in solving some test problems. Following the notation employed in Section 3, let $V$ and $V_{L R P I}$ respectively denote the option price (either European or American) and its approximation obtained using the LRPI method developed in the previous section. To measure the accuracy of the $V_{L R P I}$ method at the current time, the discrete maximum norm and the root mean square relative difference (RMSRD) have been used with the following definitions:

$$
\begin{gathered}
\operatorname{MaxError}_{L R P I}=\max _{i=0,1, \ldots, l}\left|V_{L R P I}\left(S_{i}, y_{0}, 0\right)-V\left(S_{i}, y_{0}, 0\right)\right|, \\
\operatorname{RMSRD}_{L R P I}=\frac{1}{l+1} \sqrt{\sum_{i=0}^{l}\left(\frac{V_{L R P I}\left(S_{i}, y_{0}, 0\right)-V\left(S_{i}, y_{0}, 0\right)}{V\left(S_{i}, y_{0}, 0\right)}\right)^{2}} .
\end{gathered}
$$

In MaxError $_{L R P I}$ and $\operatorname{RMSRD}_{L R P I}, S_{i}, i=0,1, . ., l$ are $l+1$ different points that will be chosen in a convenient neighborhood of the strike $E$, i.e. $S_{i} \in\left(\frac{4}{5} E, \frac{6}{5} E\right)$. For simplicity, in European and American options we set $S_{i}=(0.1 i+0.8) E$, where $i \in \Xi_{1}=\{0,1,2,3,4\}$ or $i \in \Xi_{2}=\{1,2,3\}$. Note that only in the case of the European option under SV model the exact value of $V$ is available. Therefore to the other methods we use instead the reference prices which are described in previous papers, where they have been obtained by performing an accurate (and also very time-consuming) simulation on a very refined mesh.

In the following analysis, the optimal values of the radius of the local sub-domains is selected using the figures for MaxError (or RMSRD) vs different values of $r_{Q}$ (for example see Figures 3 and 4 for SVJ model). The size of $r_{Q}$ is such that the union of these sub-domains must cover the whole global domain i.e. $\cup \Omega_{s}^{i} \subset \Omega$. It is also worth noticing that the LRPI approximation is well-defined only when $\mathbf{G}$ is non-singular or the rank of $\mathbf{P}$ equals $m$ and at least $m$ radial functions are non-zero i.e. $n>m$ for each $\mathbf{x} \in \Omega$. Therefore, to satisfy these conditions, the size of the support domain $r_{w}$ should be large enough to have sufficient number of nodes covered in $\Omega_{s}^{i}$ for every sample point $(n>m)$. In all the simulations presented in this work we use $r_{w}=l h$, where $l=1.5,2,2.5,3$, and $h$ is the distance between the nodes. Note that $h=\max (\Delta x, \Delta z)$ and also $1.5 h$ is the smallest possible choice for $r_{w}$. Figures 3 and 4 for SVJ model are considered to illustrate the effect of the radius of the local sub-domains $r_{Q}$ and the size of the support domain $r_{w}$ on our solutions. In these figures, the effect of $r_{Q}$ and $r_{w}$ on MaxError (or RMSRD) are shown. The radius of the local sub-domains $r_{Q}$ and the size of the support domain $r_{w}$ should be chosen to reduce the value of MaxError (or RMSRD). From these figures, it can be seen that in all the simulations presented in this work, the accuracy grows as the size of the support domain $r_{w}$ increases gradually. On the other hand, we know that an increase in the size of the support domain $r_{w}$, increases the CPU time computed in the approximation. Therefore, as we have experimentally checked the best value of the size of the support domain $r_{w}$ is $1.5 h$ for SV, SVJ and SVCJ models. 
Also, for all models we use $\xi_{s}=1$ and $\xi_{y}=10$. These values are chosen by trial and error such as to roughly minimize the errors on the numerical solutions.

To show the rate of convergence of the new scheme when $h \rightarrow 0$ and $\Delta t \rightarrow 0$, the values of ratio with the following formula have been reported in the tables

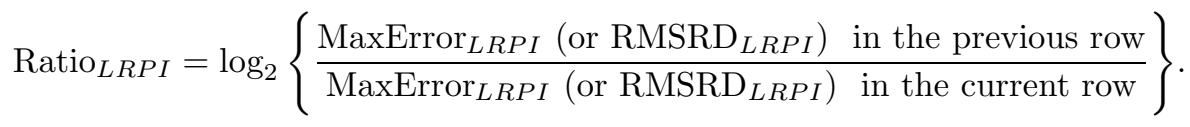

Also, the computer time required to obtain the option price using the numerical method described in previous section is denoted by $C P U$ time.

Finally, the numerical implementation and all of the executions are performable by Matlab software, alongside hardware configuration: Intel(R) Core(TM)2 Duo CPU T9550 2.66 GHz 4 GB RAM.

\subsection{Test case 1: SV models}

To demonstrate the excellent capability of the presented method, first example considers the European and American options under SV model. In particular, we consider the same test case reported in [19, 46, 66, 34, 33, 48, 65, 35], where the option and model parameters are chosen as in Table 1.

\begin{tabular}{|c|c|c|c|c|c|c|c|c|c|c|c|c|c|c|}
\hline & & $E$ & $T$ (year) & $r$ & $q$ & $\xi$ & $\eta$ & $\theta$ & $\lambda$ & $\delta$ & $\gamma$ & $\rho$ & $\rho_{j}$ & $\nu$ \\
\hline \multirow[t]{2}{*}{$\overline{\text { Test case } 1}$} & E.p [31] & 10 & 0.25 & 0.1 & - & 5 & 0.16 & 0.9 & - & - & - & 0.1 & - & - \\
\hline & A.p $[19,46,66,34,33,48,65,35]$ & 10 & 0.25 & 0.1 & - & 5 & 0.16 & 0.9 & - & - & - & 0.1 & - & - \\
\hline \multicolumn{15}{|l|}{ Test case 2} \\
\hline & E.p $[57]$ & 100 & 0.5 & 0.03 & - & 2 & 0.04 & 0.25 & 0.2 & 0.04 & -0.5 & -0.5 & - & - \\
\hline & A.p [57] & 100 & 0.5 & 0.03 & - & 2 & 0.04 & 0.25 & 0.2 & 0.04 & -0.5 & -0.5 & - & - \\
\hline & A.c $[11,17]$ & 100 & 0.5 & 0.03 & 0.05 & 2 & 0.04 & 0.4 & 5 & 0.1 & -0.005 & 0.5 & - & - \\
\hline & A.c $[11,17]$ & 100 & 0.5 & 0.03 & 0.05 & 2 & 0.04 & 0.4 & 5 & 0.1 & -0.005 & -0.5 & - & - \\
\hline & A.c $[11,60]$ & 100 & 0.5 & 0.03 & 0.05 & 2 & 0.04 & 0.25 & 0.2 & 0.4 & -0.5 & -0.5 & - & - \\
\hline \multirow{2}{*}{\multicolumn{15}{|c|}{ Test case 3}} \\
\hline & E.p [57] & 100 & 0.5 & 0.03 & - & 2 & 0.04 & 0.25 & 0.2 & 0.04 & -0.5 & -0.5 & -0.5 & 0.2 \\
\hline & A.p [57] & 100 & 0.5 & 0.03 & - & 2 & 0.04 & 0.25 & 0.2 & 0.04 & -0.5 & -0.5 & -0.5 & 0.2 \\
\hline
\end{tabular}

It should be noted that in all simulations proposed in this work, we have $\left[0, S_{\max }\right] \times\left[0, y_{\max }\right]=[0,4 E] \times$ $[0,1]$. Again we do emphasize that in the American option under SV model, the exact value of $V$ is not available. Therefore we use instead the reference prices which are described in [19, 46, 66, 34, 33, 48, 65, 35]. The reference prices for these options are given in Table 2.

Testifying the accuracy, numerical rate of convergence of the solution and CPU time with respect to the number of scattered nodes are of our special interest. To achieve this goal, we apply the local weak form meshless method by employing the radial point interpolation based on Wendland's compactly supported radial basis functions with $C^{2}, C^{4}$ and $C^{6}$ smoothness together with different choice of $N_{x}, N_{z}$ and $M$ to evaluate European and American options of this financial model. The result are given in Tables 3 and 4 . So, we derive the fast and accurate solutions that possess both properties convergence and stability from the numerical point of view. The tables given in this model obviously confirm this claim. The number of time discretization steps is set equal to nodes distributed in the volatility dimension of the asset price. As we have experimentally checked, this choice is such that in all the simulations performed the error due to the time discretization is negligible with respect to the error due to the LRPI discretization (note that in the present work we are mainly concerned with the LRPI spatial approximation). Paying attention, from these tables we observe that the accuracy grows as the number of nodes increases gradually, this fact can be understood more clear from the data shown in Tables 3 and 4 since as the number of nodes increases, we find the approximations that possess more correct digits.. Then the option price can be computed with a small financial error in a small computer time. This indicates that the numerical solution converges to the true solution as the number of nodes increases gradually. Again we do confirm that the true solution is available only in European option which is proposed in [31]. Therefore, in American option, we use instead the reference price which is described in [33], where it has been obtained by performing an accurate (and also very time-consuming) simulation on a very refined mesh.

In fact, considering Table 3 , by employing $64 \times 32$ nodal points in domain and 32 time discretization steps, European option under SV model is computed with an error of order $O\left(10^{-6}\right)$ in less than 0.01 seconds, instead, using $128 \times 64$ nodal points and 64 time discretization steps, the option price is computed 
Table 2: Reference prices used in American put option under SV model.

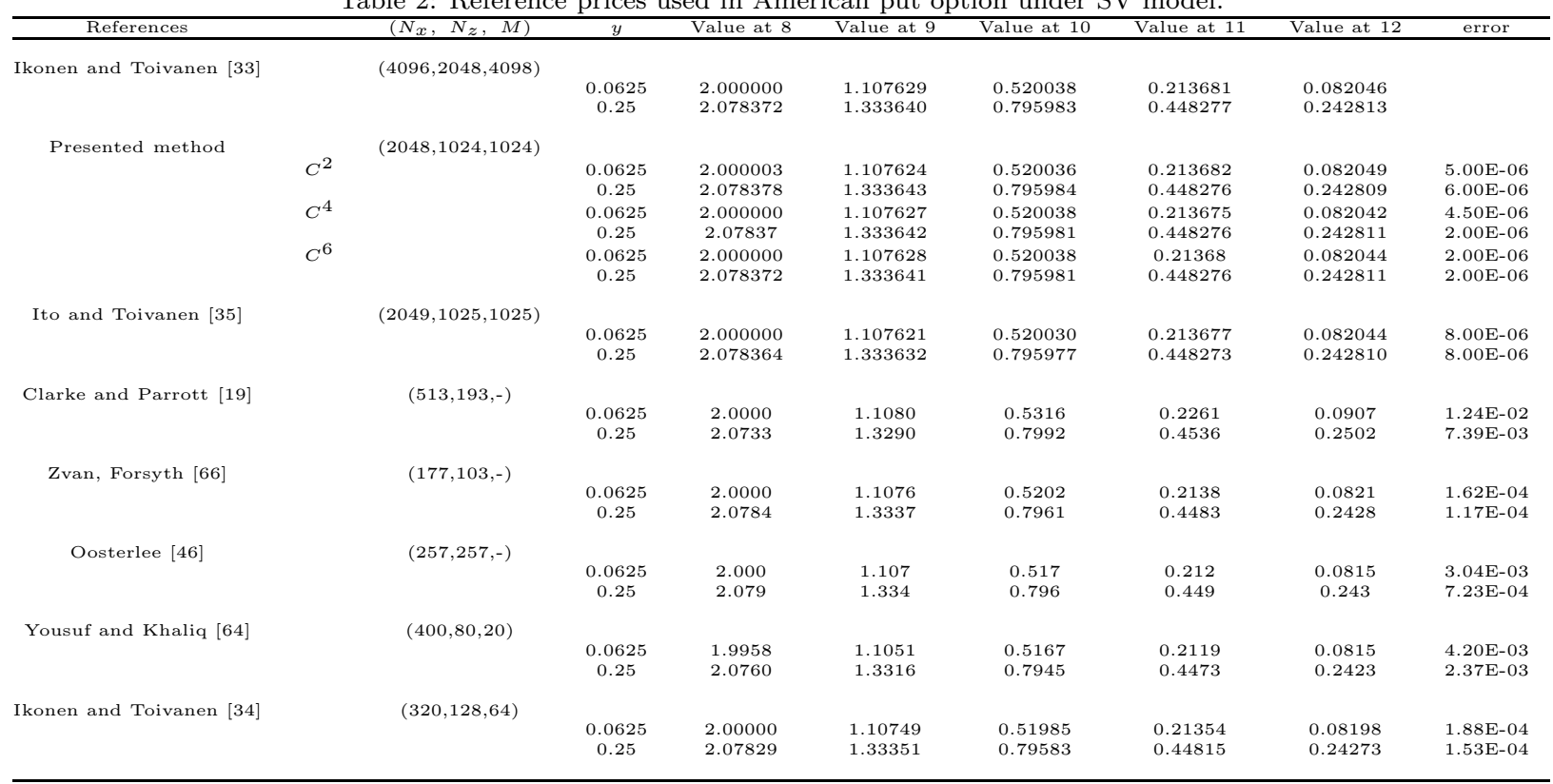

Table 3: Test case 1: European put option.

\begin{tabular}{|c|c|c|c|c|c|c|c|c|c|}
\hline \multirow[b]{2}{*}{$\left(N_{x}, N_{z}, M\right)$} & \multicolumn{3}{|c|}{$C^{2}$} & \multicolumn{3}{|c|}{$C^{4}$} & \multicolumn{3}{|c|}{$C^{6}$} \\
\hline & error & Ratio & CPU time & error & Ratio & CPU time & error & Ratio & CPU time \\
\hline$(16,8,8)$ & $4.30 \mathrm{E}-05$ & - & $<0.01$ & $2.78 \mathrm{E}-05$ & - & $<0.01$ & $2.11 \mathrm{E}-05$ & - & $<0.01$ \\
\hline$(32,16,16)$ & 1.91E-05 & 1.17 & $<0.01$ & $7.70 \mathrm{E}-06$ & 1.85 & $<0.01$ & $7.93 \mathrm{E}-06$ & 1.41 & $<0.01$ \\
\hline$(64,32,32)$ & $5.55 \mathrm{E}-06$ & 1.78 & $<0.01$ & $2.03 \mathrm{E}-06$ & 1.92 & $<0.01$ & $2.02 \mathrm{E}-06$ & 1.97 & $<0.01$ \\
\hline$(128,64,64)$ & $1.72 \mathrm{E}-06$ & 1.69 & 0.19 & $5.37 \mathrm{E}-07$ & 1.92 & 0.19 & $5.40 \mathrm{E}-07$ & 1.90 & 0.19 \\
\hline$(256,128,128)$ & $4.81 \mathrm{E}-07$ & 1.84 & 0.42 & $1.38 \mathrm{E}-07$ & 1.96 & 0.42 & $1.36 \mathrm{E}-07$ & 1.99 & 0.42 \\
\hline$(512,256,256)$ & $1.28 \mathrm{E}-07$ & 1.91 & 1.80 & $3.48 \mathrm{E}-08$ & 1.99 & 1.80 & $3.39 \mathrm{E}-08$ & 2.00 & 1.80 \\
\hline$(1024,512,512)$ & $3.37 \mathrm{E}-08$ & 1.92 & 7.01 & $8.68 \mathrm{E}-09$ & 2.00 & 7.01 & $8.42 \mathrm{E}-09$ & 2.01 & 7.01 \\
\hline$(2048,1024,1024)$ & $8.89 \mathrm{E}-09$ & 1.92 & 24.43 & $2.16 \mathrm{E}-09$ & 2.01 & 24.43 & $2.09 \mathrm{E}-09$ & 2.01 & 24.43 \\
\hline
\end{tabular}

with an error $O\left(10^{-7}\right)$ in 0.19 second and using $512 \times 256$ nodal points and 256 time discretization steps, the option price is computed with an error $O\left(10^{-8}\right)$ in 1.80 second. This means especially that the computer times necessary to perform these simulations are extremely small. Note that, for example, in Table 3, as well as in the following ones, the fact that error is approximately $10^{-6}$ means that $V_{L R P I}\left(s, y_{0}, 0\right)$ is up to at least the 6 th significant digit, equal to $V\left(s, y_{0}, 0\right)$. On the other hand, by looking at Table 4 , it can also be seen that in LRPI scheme error $O\left(10^{-3}\right), O\left(10^{-4}\right)$ and $O\left(10^{-5}\right)$ are computed using $64 \times 32,256 \times 128$ and $512 \times 256$ nodal points and $32,128,256$ time discretization steps, respectively in $0,0.33$ and 0.81 seconds. As the additional point, we can observe that the option price computed by $C^{2}, C^{4}$ and $C^{6}$ Wendland's compactly supported radial basis functions on sub-domains require the same very small CPU times to reach an accuracy of $6 \times 10^{-6}, 4.5 \times 10^{-6}$ and $2 \times 10^{-6}$ on the same number of sub-domains. Therefore, we can simply conclude that in Wendland's compactly supported radial basis functions, the accuracy grows as the smoothness order of these functions increases. On the other hand, American option price is obtained by Richardson extrapolation of the prices of two Bermudan options. For this reason and due to the fact that the maximum term (3.40) is also computed in American option, the times for American options are higher

Table 4: Test case 1: American put option.

\begin{tabular}{|c|c|c|c|c|c|c|c|c|c|}
\hline \multirow[b]{2}{*}{$\left(N_{x}, N_{z}, M\right)$} & \multicolumn{3}{|c|}{$C^{2}$} & \multicolumn{3}{|c|}{$C^{4}$} & \multicolumn{3}{|c|}{$C^{6}$} \\
\hline & error & Ratio & CPU time & error & Ratio & CPU time & error & Ratio & CPU time \\
\hline$(16,8,8)$ & $7.30 \mathrm{E}-02$ & - & $<0.01$ & $5.16 \mathrm{E}-02$ & - & $<0.01$ & $1.87 \mathrm{E}-02$ & - & $<0.01$ \\
\hline$(32,16,16)$ & $7.89 \mathrm{E}-03$ & 3.21 & $<0.01$ & $1.69 \mathrm{E}-02$ & 1.61 & $<0.01$ & $5.30 \mathrm{E}-03$ & 1.82 & $<0.01$ \\
\hline$(64,32,32)$ & $2.48 \mathrm{E}-03$ & 1.67 & $<0.01$ & $4.67 \mathrm{E}-03$ & 1.86 & $<0.01$ & $1.43 \mathrm{E}-03$ & 1.89 & $<0.01$ \\
\hline$(128,64,64)$ & $1.03 \mathrm{E}-03$ & 1.27 & 0.33 & $1.21 \mathrm{E}-03$ & 1.95 & 0.33 & $3.83 \mathrm{E}-04$ & 1.90 & 0.33 \\
\hline$(256,128,128)$ & $2.68 \mathrm{E}-04$ & 1.94 & 0.81 & $3.07 \mathrm{E}-04$ & 1.98 & 0.81 & $1.02 \mathrm{E}-04$ & 1.91 & 0.81 \\
\hline$(512,256,256)$ & $7.09 \mathrm{E}-05$ & 1.92 & 2.70 & $7.73 \mathrm{E}-05$ & 1.99 & 2.70 & $3.13 \mathrm{E}-05$ & 1.70 & 2.70 \\
\hline$(1024,512,512)$ & $1.98 \mathrm{E}-05$ & 1.84 & 11.14 & $1.92 \mathrm{E}-05$ & 2.01 & 11.14 & $7.94 \mathrm{E}-06$ & 1.98 & 11.14 \\
\hline$(2048,1024,1024)$ & $6.00 \mathrm{E}-06$ & 1.72 & 35.68 & $4.50 \mathrm{E}-06$ & 2.09 & 35.68 & $2.00 \mathrm{E}-06$ & 1.99 & 35.68 \\
\hline
\end{tabular}


on the same grids. The next issue is examination of the numerical rate of convergence. As the final look at the numerical results in Tables 3 and 4, we can see rate of convergence of LRPI is 2.

Table 5: Comparison of the pseudospectral method in [45] and the proposed method with reference price values for $s_{0}=$ $8,9,10,11,12$ and $y=0.062$ and 0.25 in American put option under SV model. The other parameters used are $E=10$, $T=0.25, r=0.1, \xi=5, \eta=0.16, \theta=0.9$ and $\rho=0.1$.

\begin{tabular}{|c|c|c|c|c|c|}
\hline$y$ & $S$ & Reference & price $\left(N_{x}=4096, N_{z}=2048\right)[33]$ & $\operatorname{Error}_{L R P I}\left(N_{x}=16, N_{z}=8\right)$ & Errorpseudospectral $\left(N_{x}=25, N_{z}=15\right)$ [45] \\
\hline \multirow[t]{5}{*}{0.0625} & 8 & & 2.000000 & $1.53 \mathrm{E}-02$ & $9.84 \mathrm{E}-02$ \\
\hline & 9 & & 1.107629 & $1.12 \mathrm{E}-02$ & $4.47 \mathrm{E}-02$ \\
\hline & 10 & & 0.520038 & $1.74 \mathrm{E}-02$ & $2.22 \mathrm{E}-02$ \\
\hline & 11 & & 0.213681 & $1.67 \mathrm{E}-02$ & $6.35 \mathrm{E}-02$ \\
\hline & 12 & & 0.082046 & $1.02 \mathrm{E}-02$ & $3.25 \mathrm{E}-03$ \\
\hline \multirow[t]{5}{*}{0.25} & 8 & & 2.078372 & $1.11 \mathrm{E}-02$ & $1.46 \mathrm{E}-02$ \\
\hline & 9 & & 1.333640 & $1.35 \mathrm{E}-02$ & $8.76 \mathrm{E}-02$ \\
\hline & 10 & & 0.795983 & $1.87 \mathrm{E}-02$ & $9.86 \mathrm{E}-02$ \\
\hline & 11 & & 0.448277 & $9.83 \mathrm{E}-03$ & $2.16 \mathrm{E}-02$ \\
\hline & 12 & & 0.242813 & $8.77 \mathrm{E}-03$ & $1.83 \mathrm{E}-02$ \\
\hline
\end{tabular}

We end this section by comparing our proposed method to a pseudospectral method to solve SV model for a American put option. In [45], this was one of the methods presented for this problem. The authors of [45] simulated this model using a combination of the pseudospectral collocation method and the Laplace transforms method. To approximate the two dimensional PDE, they constructed a grid which was the tensor product of the two grids, each of which was based on the Chebyshev points in the two spacial directions. In the solution of the SV model, the computational domain $\left[0, s_{\max }\right] \times\left[0, y_{\max }\right]=[0,4 E] \times[0,1]$ and $\left[0, s_{\max }\right] \times\left[0, y_{\max }\right]=[0,2 E] \times[0,1]$ are used in LRPI and pseudospectral methods, respectively. The value of $M$ is for LRPI method manually determined to be the smallest value that does not influence the total error in the solution. Note that to measure the accuracy of the $V_{L R P I}$ and $V_{\text {pseudospectral }}$ methods at the current time, the absolute error the methods and the reference price values reported in [33] have been used i.e. Error $_{L R P I}=\left|V_{L R P I}-V_{\text {Reference price }}\right|$ and Error $_{L R P I}=\left|V_{\text {pseudospectral }}-V_{\text {Reference price }}\right|$.

\subsection{Test case 2: SVJ models}

This example, illustrates the applicability of the proposed method to five different option pricing problem under SVJ model with the following specifications

1. European put option presented in [57],

2. American put option presented in [57],

3. American call option with positive correlation presented in [17, 11],

4. American call option with negative correlation presented in [17, 11],

5. American call option presented in [60, 11],

where the option and model parameters are chosen as in Table 1.

First, let us consider the European and American put options under the Bates models considered by Bates [12]. As was done in [57], for the initial $S_{0}$ we consider three different values $S_{i}$ in a convenient neighborhood of the strike $E$ so that in their the error is greater than elsewhere, i.e. $i \in \Xi_{2}$. Moreover we set $y_{0}=0.04$. Besides, as was done in Test case 1 , we suppose that $\left[0, S_{\max }\right] \times\left[0, y_{\max }\right]=[0,4 E] \times[0,1]$.

Similar to the American SV model in Test case 1, here the true price is not available (neither in the European nor in the American case), thus the true price is replaced with a reference price listed in Tables 6 and 7, which have been obtained in [57] using the projected algebraic multigrid (PAMG) method on an extremely fine grid with 4097, 2049 and 513 nodes in $s, y$ and $t$ directions, respectively.

Table 6: Reference prices used in European put option under SVJ model using model parameters presented in [57].

\begin{tabular}{|c|c|c|c|c|c|}
\hline \multirow{5}{*}{$\begin{array}{c}\text { References } \\
\text { Salmi }[57] \\
\text { Proposed method }\end{array}$} & & $\left(N_{x}, N_{z}, M\right)$ & Value at 90 & Value at 100 & Value at 110 \\
\hline & & $(4097,2049,513)$ & & & \\
\hline & $C^{2}$ & $(2048,1024,1024)$ & 11.302908 & 6.589891 & 4.191446 \\
\hline & & $(2048,1024,1024)$ & 11.302912 & 6.589888 & 4.191449 \\
\hline & $C^{6}$ & $(2048,1024,1024)$ & 11.302913 & 6.589887 & 4.191449 \\
\hline
\end{tabular}

Applying the LRPI method based on Wendland's compactly supported radial basis functions with $C^{2}, C^{4}$ and $C^{6}$ smoothness presented in this paper, we obtain the results tabulated in Tables 8 and 9 . It is seen from the tabulated results that the approximations of option price are improved by increasing number of 
Table 7: Reference prices used in American put option under SVJ model using model parameters presented in [57].

\begin{tabular}{|c|c|c|c|c|c|}
\hline References & & $\left(N_{x}, N_{z}, M\right)$ & Value at 90 & Value at 100 & Value at 110 \\
\hline \multirow{4}{*}{ Proposed method } & & $\frac{x, 2 z,}{(4097,2049,513)}$ & 11.619920 & 6.714240 & 4.261583 \\
\hline & $C^{2}$ & $(2048,1024,1024)$ & 11.619914 & 6.714249 & 4.261568 \\
\hline & $C^{4}$ & $(2048,1024,1024)$ & 11.619914 & 6.714247 & 4.261571 \\
\hline & $C^{6}$ & $(2048,1024,1024)$ & 11.619916 & 6.714247 & 4.261575 \\
\hline
\end{tabular}

sub-domains and time discretization steps. Thus, the numerical convergence of the solution is obtained. Indeed, it can be seen that the LRPI scheme provides very fast approximation for this model.

Comparing the profiles of approximations, one can conclude that similar to the above discussion, LRPI method with $C^{6}$ smoothness functions is more accurate than the proposed method using Wendland's radial basis functions with lower smoothness degree, but smoothness degree of basis functions is no effect on CPU time of presented algorithm.

Table 8: Test case 2: European put option under SVJ model using model parameters presented in [57].

\begin{tabular}{|c|c|c|c|c|c|c|c|c|c|}
\hline \multirow[b]{2}{*}{$\left(N_{x}, N_{z}, M\right)$} & \multicolumn{3}{|c|}{$C^{2}$} & \multicolumn{3}{|c|}{$C^{4}$} & \multicolumn{3}{|c|}{$C^{6}$} \\
\hline & RMSRD & Ratio & CPU time & RMSRD & Ratio & CPU time & RMSRD & Ratio & CPU time \\
\hline$(16,8,8)$ & $6.72 \mathrm{E}-03$ & - & 0.04 & $1.08 \mathrm{E}-02$ & - & 0.04 & $7.20 \mathrm{E}-03$ & - & 0.04 \\
\hline$(32,16,16)$ & $3.07 \mathrm{E}-03$ & 1.15 & 0.11 & $3.50 \mathrm{E}-03$ & 1.62 & 0.11 & $2.11 \mathrm{E}-03$ & 1.77 & 0.11 \\
\hline$(64,32,32)$ & $1.22 \mathrm{E}-03$ & 1.33 & 0.30 & $9.51 \mathrm{E}-04$ & 1.88 & 0.30 & $8.18 \mathrm{E}-04$ & 1.37 & 0.30 \\
\hline$(128,64,64)$ & $2.29 \mathrm{E}-04$ & 2.42 & 0.55 & $2.53 \mathrm{E}-04$ & 1.91 & 0.55 & $2.27 \mathrm{E}-04$ & 1.85 & 0.55 \\
\hline$(256,128,128)$ & $6.91 \mathrm{E}-05$ & 1.73 & 1.29 & $6.59 \mathrm{E}-05$ & 1.94 & 1.29 & $6.53 \mathrm{E}-05$ & 1.80 & 1.29 \\
\hline$(512,256,256)$ & $1.80 \mathrm{E}-05$ & 1.94 & 4.17 & $1.67 \mathrm{E}-05$ & 1.98 & 4.17 & $1.63 \mathrm{E}-05$ & 2.00 & 4.17 \\
\hline$(1024,512,512)$ & $4.85 \mathrm{E}-06$ & 1.89 & 16.33 & $4.33 \mathrm{E}-06$ & $\begin{array}{l}1.95 \\
1.95\end{array}$ & 16.33 & $4.03 \mathrm{E}-06$ & $\begin{array}{l}2.00 \\
2.01\end{array}$ & 16.33 \\
\hline$(2048,1024,1024)$ & $1.59 \mathrm{E}-06$ & 1.61 & 58.46 & $1.06 \mathrm{E}-06$ & 2.03 & 58.46 & $1.00 \mathrm{E}-06$ & 2.01 & 58.46 \\
\hline
\end{tabular}

Table 9: Test case 2: American put option under SVJ model using model parameters presented in [57].

\begin{tabular}{|c|c|c|c|c|c|c|c|c|c|}
\hline \multirow[b]{2}{*}{$\left(N_{x}, N_{z}, M\right)$} & \multicolumn{3}{|c|}{$C^{2}$} & \multicolumn{3}{|c|}{$C^{4}$} & \multicolumn{3}{|c|}{$C^{6}$} \\
\hline & RMSRD & Ratio & CPU time & RMSRD & Ratio & CPU time & RMSRD & Ratio & CPU time \\
\hline$(16,8,8)$ & $9.91 \mathrm{E}-03$ & - & 0.07 & $2.55 \mathrm{E}-02$ & - & 0.07 & $7.48 \mathrm{E}-03$ & - & 0.07 \\
\hline$(32,16,16)$ & $1.31 \mathrm{E}-03$ & 2.92 & 0.19 & $7.68 \mathrm{E}-03$ & 1.73 & 0.19 & $2.61 \mathrm{E}-03$ & 1.52 & 0.19 \\
\hline$(64,32,32)$ & $4.58 \mathrm{E}-04$ & 1.52 & 0.49 & $2.19 \mathrm{E}-03$ & 1.81 & 0.49 & $7.99 \mathrm{E}-04$ & 1.71 & 0.49 \\
\hline$(128,64,64)$ & $1.81 \mathrm{E}-04$ & 1.34 & 0.81 & $8.20 \mathrm{E}-04$ & 1.42 & 0.81 & $3.09 \mathrm{E}-04$ & 1.37 & 0.81 \\
\hline$(256,128,128)$ & $7.98 \mathrm{E}-05$ & 1.18 & 1.92 & $2.26 \mathrm{E}-04$ & 1.86 & 1.92 & $8.17 \mathrm{E}-05$ & 1.92 & 1.92 \\
\hline$(512,256,256)$ & $2.26 \mathrm{E}-05$ & 1.82 & 6.53 & $6.16 \mathrm{E}-05$ & 1.88 & 6.53 & $2.33 \mathrm{E}-05$ & 1.81 & 6.53 \\
\hline$(1024,512,512)$ & $6.78 \mathrm{E}-06$ & 1.74 & 27.11 & $6.99 \mathrm{E}-06$ & 3.14 & 27.11 & $5.48 \mathrm{E}-06$ & 2.09 & 27.11 \\
\hline$(2048,1024,1024)$ & $2.19 \mathrm{E}-06$ & 1.63 & 92.89 & $1.76 \mathrm{E}-06$ & 1.99 & 92.89 & $1.26 \mathrm{E}-06$ & 2.12 & 92.89 \\
\hline
\end{tabular}

Here, due to the fact that it is almost impossible to explain obtained numerical results in all cases of nodal points and time discretization steps exactly in practice, to satisfy our curiosity we explain a bit more about some of the error values using $32 \times 16,128 \times 64$ and $512 \times 256$ nodal points and 16, 64 and 256 time discretization steps with CPU times.

To clarify even more, for example, using $32 \times 16$ nodal points in domain and 16 time discretization steps, European option under SVJ model is computed with an error of order $O\left(10^{-3}\right)$ in only 0.11 second, instead, using $128 \times 64$ nodal points and 64 time discretization steps, the option price is computed with an error $O\left(10^{-4}\right)$ in 0.55 second and using $512 \times 256$ nodal points and 256 time discretization steps, the option price is computed with an error $O\left(10^{-5}\right)$ in 4.17 seconds. On the other hand, by looking at Table 9 , it can also be seen that in LRPI scheme error $O\left(10^{-3}\right), O\left(10^{-4}\right)$ and $O\left(10^{-5}\right)$ are computed using $32 \times 16,128 \times 64$ and $512 \times 256$ nodal points and 16, 64 and 256 time discretization steps, respectively in $0.19,0.81$ and 6.53 seconds. It should be noted that Tables 8 and 9 have much higher times than Tables 3 and 4 for the same grids as expected. It is clear that this is due to the computation of the integral part.

Worthy of being considered at the end, here similar to Test case 1, the rate of convergence of LRPI is 2 (either in the European or in the American case).

Second, we consider the same test-case presented by Chiarella et al. [17] and Ballestra et al. [11], in which the model parameters and the option's data are chosen as in Table 1. Again, we set $y_{0}=0.04, s=S_{i}$ so that $i \in \Xi_{1}$, and $\left[0, S_{\max }\right] \times\left[0, y_{\max }\right]=[0,4 E] \times[0,1]$. It should be noted that the model is considered using positive and negative correlation. The references value of option pricing is presented in Table 10. In this table, the true price have been obtained in [17] using a finite difference approximation on an extremely fine mesh with 6000, 3000 and 1000 points in $s, y$ and $t$ directions, respectively.

The results of implementing the problem by utilizing the present method with various number of subdomains and time discretization steps are shown in Tables 11 and 12. 
Table 10: Reference prices used in American call option under SVJ model using model parameters presented in [17, 11].

\begin{tabular}{|c|c|c|c|c|c|c|c|c|}
\hline & References & & $\left(N_{x}, N_{z}, M\right)$ & Value at 80 & Value at 90 & Value at 100 & Value at 110 & Value at 120 \\
\hline \multirow[t]{5}{*}{$\rho=0.5$} & Chiarella et. al. $[17]$ & & $(6000,3000,1000)$ & 1.4843 & 3.7145 & 7.7027 & 13.6722 & 21.3653 \\
\hline & Ballestra and Sgarra [11] & & $(250,200,20)$ & 1.4849 & 3.7159 & 7.7044 & 13.6735 & 21.3661 \\
\hline & Proposed method & $C^{2}$ & $(2048,1024,1024)$ & 1.4843 & 3.7145 & 7.7027 & 13.6722 & 21.3653 \\
\hline & & $C^{4}$ & $(2048,1024,1024)$ & 1.4843 & 3.7145 & 7.7027 & 13.6722 & 21.3653 \\
\hline & & $C^{6}$ & $(2048,1024,1024)$ & 1.4843 & 3.7145 & 7.7027 & 13.6722 & 21.3653 \\
\hline \multirow{6}{*}{$\rho=-0.5$} & & & & & & & & \\
\hline & Chiarella et. al. [17] & & $(6000,3000,1000)$ & 1.1359 & 3.3532 & 7.5970 & 13.8830 & 21.7186 \\
\hline & Ballestra and Sgarra [11] & & $(250,200,20)$ & 1.1356 & 3.3537 & 7.5986 & 13.8852 & 21.7209 \\
\hline & Proposed method & $C^{2}$ & $(2048,1024,1024)$ & 1.1359 & 3.3532 & 7.5970 & 13.8830 & 21.7186 \\
\hline & & $C^{2}$ & $(2048,1024,1024)$ & 1.1359 & 3.3532 & 7.5970 & 13.8830 & 21.7186 \\
\hline & & $C^{2}$ & $(2048,1024,1024)$ & 1.1359 & 3.3532 & 7.5970 & 13.8830 & 21.7186 \\
\hline
\end{tabular}

Table 11: Test case 2: American call option under SVJ model using model parameters presented in [17, 11], $\rho=0.5$.

\begin{tabular}{|c|c|c|c|c|c|c|c|c|c|}
\hline \multirow[b]{2}{*}{$\left(N_{x}, N_{z}, M\right)$} & \multicolumn{3}{|c|}{$C^{2}$} & \multicolumn{3}{|c|}{$C^{4}$} & \multicolumn{3}{|c|}{$C^{6}$} \\
\hline & RMSRD & Ratio & CPU time & RMSRD & Ratio & CPU time & RMSRD & Ratio & CPU time \\
\hline$(16,8,8)$ & $2.56 \mathrm{E}-02$ & - & 0.07 & $5.47 \mathrm{E}-02$ & - & 0.07 & $5.86 \mathrm{E}-02$ & - & 0.07 \\
\hline$(32,16,16)$ & $7.10 \mathrm{E}-03$ & 1.85 & 0.19 & $1.92 \mathrm{E}-02$ & 1.51 & 0.19 & $2.48 \mathrm{E}-02$ & 1.24 & 0.19 \\
\hline$(64,32,32)$ & $2.36 \mathrm{E}-03$ & 1.59 & 0.49 & $5.54 \mathrm{E}-03$ & 1.79 & 0.49 & $6.74 \mathrm{E}-03$ & 1.88 & 0.49 \\
\hline$(128,64,64)$ & $8.52 \mathrm{E}-04$ & 1.47 & 0.81 & $1.57 \mathrm{E}-03$ & 1.82 & 0.81 & $1.77 \mathrm{E}-03$ & 1.93 & 0.81 \\
\hline$(256,128,128)$ & $2.30 \mathrm{E}-04$ & 1.89 & 1.92 & $4.24 \mathrm{E}-04$ & 1.89 & 1.92 & $4.54 \mathrm{E}-04$ & 1.96 & 1.92 \\
\hline$(512,256,256)$ & $9.67 \mathrm{E}-05$ & 1.25 & 6.53 & $1.11 \mathrm{E}-04$ & 1.93 & 6.53 & $1.12 \mathrm{E}-04$ & 2.02 & 6.53 \\
\hline$(1024,512,512)$ & $3.04 \mathrm{E}-05$ & 1.67 & 27.11 & $2.81 \mathrm{E}-05$ & 1.98 & 27.11 & $2.67 \mathrm{E}-05$ & 2.07 & 27.11 \\
\hline$(2048,1024,1024)$ & $9.11 \mathrm{E}-06$ & 1.74 & 9289 & $7.07 \mathrm{E}-06$ & 1.99 & 9289 & $6.23 \mathrm{E}-06$ & 2.10 & 92.89 \\
\hline
\end{tabular}

Overall, as already pointed out, following the numerical findings in the different errors, convinces us that the error of the proposed techniques decrease very rapidly as the number of sub-domains in domain and time discretization steps increase. In fact, for example, the price of the American option can be computed with 3 and 4 correct significants in only 0.49 and 1.92 seconds, respectively which is excellent and very fast. We emphasize that the ratio shown in Tables 11 and 12 are second order.

As the last experiment, we wish to find an approximation for the American call option under SVJ model considered in [60] and [11]. Assume that $y_{0}=0.04, s=S_{i}$ so that $i \in \Xi_{1}$, and $\left[0, S_{\max }\right] \times\left[0, y_{\max }\right]=$ $[0,4 E] \times[0,1]$. The model parameters and option data are set as in Table 1 . What will be used in this model to true solution is presented in Table 13, where they have been obtained by performing an accurate (and also very time-consuming) simulation on a very refined mesh.

For the sake of brevity, here we only show the Table 14 .

\subsection{Test case 3: SVCJ models}

As the last test case, we aim to study the options under SVCJ model with the parameters and data which are presented as in Table 1 . Indeed, we get $y_{0}=0.04, s=S_{i}$ so that $i \in \Xi_{2}$, and $\left[0, S_{\max }\right] \times\left[0, y_{\max }\right]=$ $[0,4 E] \times[0,1]$. The references prices to European and American options are tabulated in Table 15, which have been obtained in [57] using the projected algebraic multigrid (PAMG) method on an extremely fine grid with 4097, 2049 and 513 nodes in $s, y$ and $t$ directions, respectively.

Implementing the proposed numerical technique produce the outcomes given by Tables 16 and 17. These tables show the numerically identified solutions for option pricing in European and American option models, respectively.

It is seen from Tables 16 and 17 that the approximations are improved by increasing the number of nodes and time discretization steps, since the RMSRD values tend to zero more quickly as the node distributions increase, which confirms convergence property of the proposed method agian. Once again, we wish to state the numerical rate of convergence in the context of this test case. It can be seen that similar to

Table 12: Test case 2: American call option under SVJ model using model parameters presented in [17, 11], $\rho=-0.5$.

\begin{tabular}{|c|c|c|c|c|c|c|c|c|c|}
\hline \multirow[b]{2}{*}{$\left(N_{x}, N_{z}, M\right)$} & \multicolumn{3}{|c|}{$C^{2}$} & \multicolumn{3}{|c|}{$C^{4}$} & \multicolumn{3}{|c|}{$C^{6}$} \\
\hline & RMSRD & Ratio & CPU time & RMSRD & Ratio & CPU time & RMSRD & Ratio & CPU time \\
\hline$(16,8,8)$ & $4.58 \mathrm{E}-02$ & - & 0.07 & $6.24 \mathrm{E}-02$ & - & 0.07 & $1.99 \mathrm{E}-02$ & - & 0.07 \\
\hline$(32,16,16)$ & $1.63 \mathrm{E}-02$ & 1.49 & 0.19 & $1.88 \mathrm{E}-02$ & 1.73 & 0.19 & $7.82 \mathrm{E}-03$ & 1.35 & 0.19 \\
\hline$(64,32,32)$ & $5.70 \mathrm{E}-03$ & 1.52 & 0.49 & $5.08 \mathrm{E}-03$ & 1.89 & 0.49 & $2.14 \mathrm{E}-03$ & 1.87 & 0.49 \\
\hline$(128,64,64)$ & $1.79 \mathrm{E}-03$ & 1.67 & 0.81 & $1.37 \mathrm{E}-03$ & 1.89 & 0.81 & $6.31 \mathrm{E}-04$ & 1.76 & 0.81 \\
\hline$(256,128,128)$ & $4.74 \mathrm{E}-04$ & 1.92 & 1.92 & $3.66 \mathrm{E}-04$ & 1.91 & 1.92 & $1.60 \mathrm{E}-04$ & 1.98 & 1.92 \\
\hline$(512,256,256)$ & $1.42 \mathrm{E}-04$ & 1.74 & 6.53 & $9.54 \mathrm{E}-05$ & 1.94 & 6.53 & $3.97 \mathrm{E}-05$ & 2.01 & 6.53 \\
\hline$(1024,512,512)$ & $3.86 \mathrm{E}-05$ & 1.88 & 27.11 & $2.43 \mathrm{E}-05$ & 1.97 & 27.11 & $9.86 \mathrm{E}-06$ & 2.01 & 27.11 \\
\hline$(2048,1024,1024)$ & $1.02 \mathrm{E}-05$ & 1.92 & 92.89 & $6.16 \mathrm{E}-06$ & 1.98 & 92.89 & $2.43 \mathrm{E}-06$ & 2.02 & 92.89 \\
\hline
\end{tabular}


Table 13: Reference prices used in American call option under SVJ model using model parameters presented in [60, 11].

\begin{tabular}{|c|c|c|c|c|c|c|c|}
\hline References & & $\left(N_{x}, N_{z}, M\right)$ & Value at 80 & Value at 90 & Value at 100 & Value at 110 & Value at 120 \\
\hline Toivanen [60] & & $(4096,2048,512)$ & 0.328526 & 2.109397 & 6.711622 & 13.749337 & 22.143307 \\
\hline Ballestra and Sgarra [11] & & $(250,200,20)$ & 0.328446 & 2.10875 & 6.711854 & 13.747836 & 22.137798 \\
\hline \multirow{3}{*}{ Proposed method } & $C^{2}$ & $(2048,1024,1024)$ & 0.328522 & 2.10939 & 6.711625 & 13.749343 & 22.143314 \\
\hline & $C^{4}$ & $(2048,1024,1024)$ & 0.328524 & 2.109393 & 6.711623 & 13.749339 & 22.143310 \\
\hline & $C^{6}$ & $(2048,1024,1024)$ & 0.328524 & 2.109394 & 6.711623 & 13.749339 & 22.143308 \\
\hline
\end{tabular}

Table 14: Test case 2: American call option under SVJ model using model parameters presented in [60, 11].

\begin{tabular}{|c|c|c|c|c|c|c|c|c|c|}
\hline \multirow[b]{2}{*}{$\left(N_{x}, N_{z}, M\right)$} & \multicolumn{3}{|c|}{$C^{2}$} & \multicolumn{3}{|c|}{$C^{4}$} & \multicolumn{3}{|c|}{$C^{6}$} \\
\hline & RMSRD & Ratio & CPU time & RMSRD & Ratio & CPU time & RMSRD & Ratio & CPU time \\
\hline$(16,8,8)$ & $1.61 \mathrm{E}-02$ & - & 0.07 & $2.25 \mathrm{E}-02$ & - & 0.07 & $5.20 \mathrm{E}-02$ & - & 0.07 \\
\hline$(32,16,16)$ & $5.61 \mathrm{E}-03$ & 1.52 & 0.19 & $8.70 \mathrm{E}-03$ & 1.37 & 0.19 & $6.41 \mathrm{E}-03$ & 3.02 & 0.19 \\
\hline$(64,32,32)$ & $1.89 \mathrm{E}-03$ & 1.57 & 0.49 & $2.48 \mathrm{E}-03$ & 1.81 & 0.49 & $1.88 \mathrm{E}-03$ & 1.77 & 0.49 \\
\hline$(128,64,64)$ & $6.25 \mathrm{E}-04$ & 1.60 & 0.81 & $6.79 \mathrm{E}-04$ & 1.87 & 0.81 & $5.41 \mathrm{E}-04$ & 1.80 & 0.81 \\
\hline$(256,128,128)$ & $1.87 \mathrm{E}-04$ & 1.74 & 1.92 & $1.82 \mathrm{E}-04$ & 1.90 & 1.92 & $1.48 \mathrm{E}-04$ & 1.87 & 1.92 \\
\hline$(512,256,256)$ & $7.24 \mathrm{E}-05$ & 1.37 & 6.53 & $4.49 \mathrm{E}-05$ & 2.02 & 6.53 & $4.08 \mathrm{E}-05$ & 1.86 & 6.53 \\
\hline$(1024,512,512)$ & $1.98 \mathrm{E}-05$ & 1.87 & 27.11 & $1.13 \mathrm{E}-05$ & 1.99 & 27.11 & $1.07 \mathrm{E}-05$ & 1.93 & 27.11 \\
\hline$(2048,1024,1024)$ & $5.65 \mathrm{E}-06$ & 1.81 & 92.89 & $2.85 \mathrm{E}-06$ & 1.99 & 92.89 & $2.80 \mathrm{E}-06$ & 1.94 & 92.89 \\
\hline
\end{tabular}

the SV and SVJ models in two previous test cases, we obtain Ratio=2 by applying the LRPI method based on Wendland's compactly supported radial basis functions with $C^{2}, C^{4}$ and $C^{6}$ smoothness. The approximations are exhibited in Tables 16 and 17 to verify this fact. Putting all these things together, we conclude that the numerical methods proposed in this paper are accurate, convergence and fast.

\section{Conclusions}

During the last decade, many works have been done to find modifications of classical Black-Scholes model to satisfy these phenomena in financial markets such as the models with stochastic volatility (SV), stochastic volatility models with jumps (SVJ), and stochastic volatility models with jumps in returns and volatility (SVCJ). Hence, an analytical solution for pricing these options is impossible. Therefore, to solve these problems, we need to have a powerful computational method. For the first time in mathematical financial field, we proposed the local weak form meshless methods for option pricing under SV, SVJ and SVCJ models; especially in this paper we focused on one of their scheme named local radial point interpolation (LRPI), based on Wendland's compactly supported radial basis functions (WCS-RBFs) with $C^{6}, C^{4}$ and $C^{2}$ smoothness degrees.

Overall the numerical achievements that should be highlighted here are as follows:

(1) The price of American option is computed by Richardson extrapolation of the price of Bermudan option. In essence the Richardson extrapolation reduces the free boundary problem and linear complementarity problem to a fixed boundary problem, which is much simpler to solve. Thus, instead of describing the aforementioned linear complementarity problem or penalty method, we directly focus our attention on the partial differential equation satisfied by the price of a Bermudan option which is faster and more accurate than other methods.

(2) The infinite space domain $\mathbb{R}^{+} \times \mathbb{R}^{+}$is truncated to $\left[0, S_{\text {max }}\right] \times\left[0, y_{\max }\right]$ in SVJ and SVCJ models, with the sufficiently large values $S_{\max }$ and $y_{\max }$ to avoid an unacceptably large truncation error. The options' payoffs considered in this paper are non-smooth functions, in particular their derivatives, are discontinuous at the strike price. Therefore, to reduce as much as possible the losses of accuracy, the points of the trial functions are concentrated on a spatial region close to the strike prices. So, we employ the change of variables proposed by Clarke and Parrott [20].

Table 15: Reference prices under SVCJ model using model parameters presented in Test case 3.

\begin{tabular}{|c|c|c|c|c|c|c|}
\hline & References & & $\left(N_{x}, N_{z}, M\right)$ & Value at 90 & Value at 100 & Value at 110 \\
\hline \multirow[t]{4}{*}{ European option } & Salmi [57] & & $(4097.2049 .513)$ & 11.134438 & 6.609162 & 4.342956 \\
\hline & Proposed method & $C^{2}$ & $(2048,1024,1024)$ & 11.134424 & 6.609173 & 4.342972 \\
\hline & & $C^{4}$ & $(2048,1024,1024)$ & 11.134429 & 6.609169 & 4.342967 \\
\hline & & $C^{6}$ & $(2048,1024,1024)$ & 11.134433 & 6.609167 & 4.342964 \\
\hline \multirow[t]{4}{*}{ American option } & Salmi $[57]$ & & $(4097,2049,513)$ & 11.561620 & 6.780527 & 4.442032 \\
\hline & Proposed method & $C^{2}$ & $(2048,1024,1024)$ & 11.561601 & 6.780541 & 4.442047 \\
\hline & & $C^{4}$ & $(2048,1024,1024)$ & 11.561611 & 6.780536 & 4.442044 \\
\hline & & $C^{6}$ & $(2048,1024,1024)$ & 11.561615 & 6.780533 & 4.442042 \\
\hline
\end{tabular}



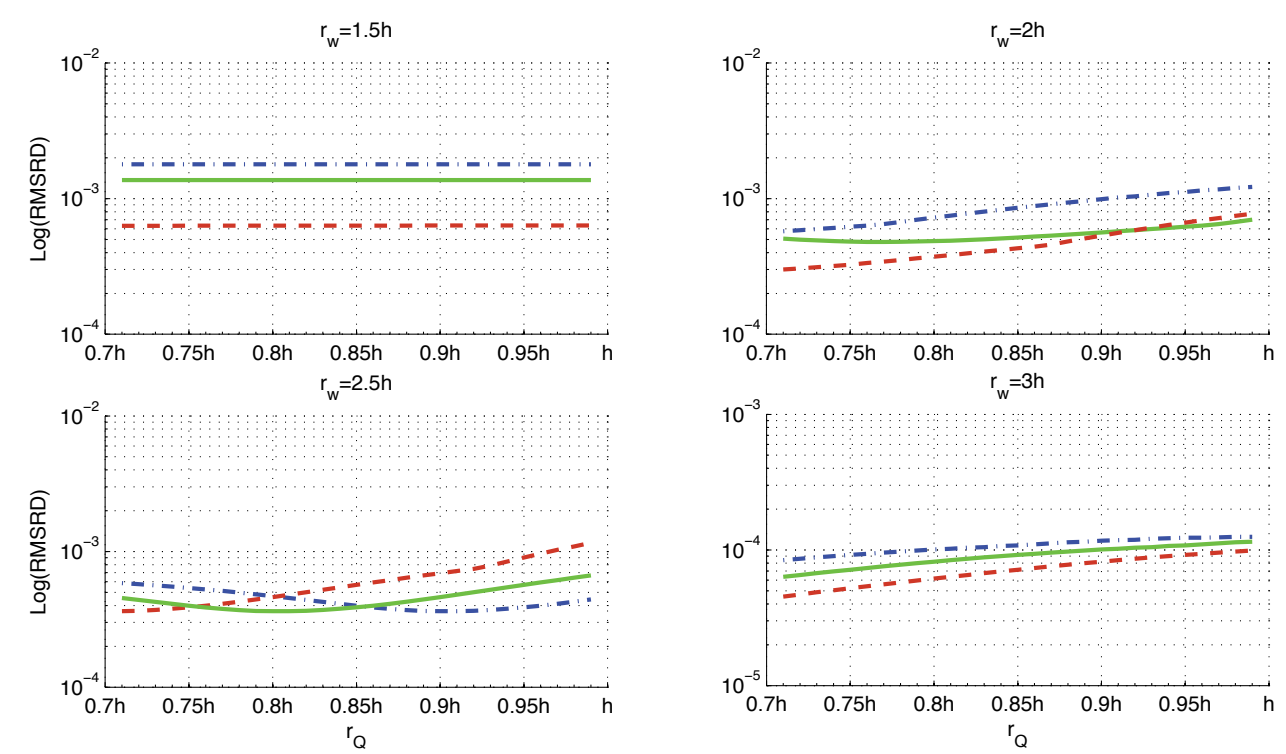

- = ' wCS-RBFs with $C^{2}$ smoothness degree $\quad$ WCS-RBFs with $C^{4}$ smoothness degree $=--=$ wCS-RBFs with $C^{6}$ smoothness degree

Figure 3: Test case 2: American call option under SVJ model using model parameters presented in [17, 11], $\rho=-0.5$.
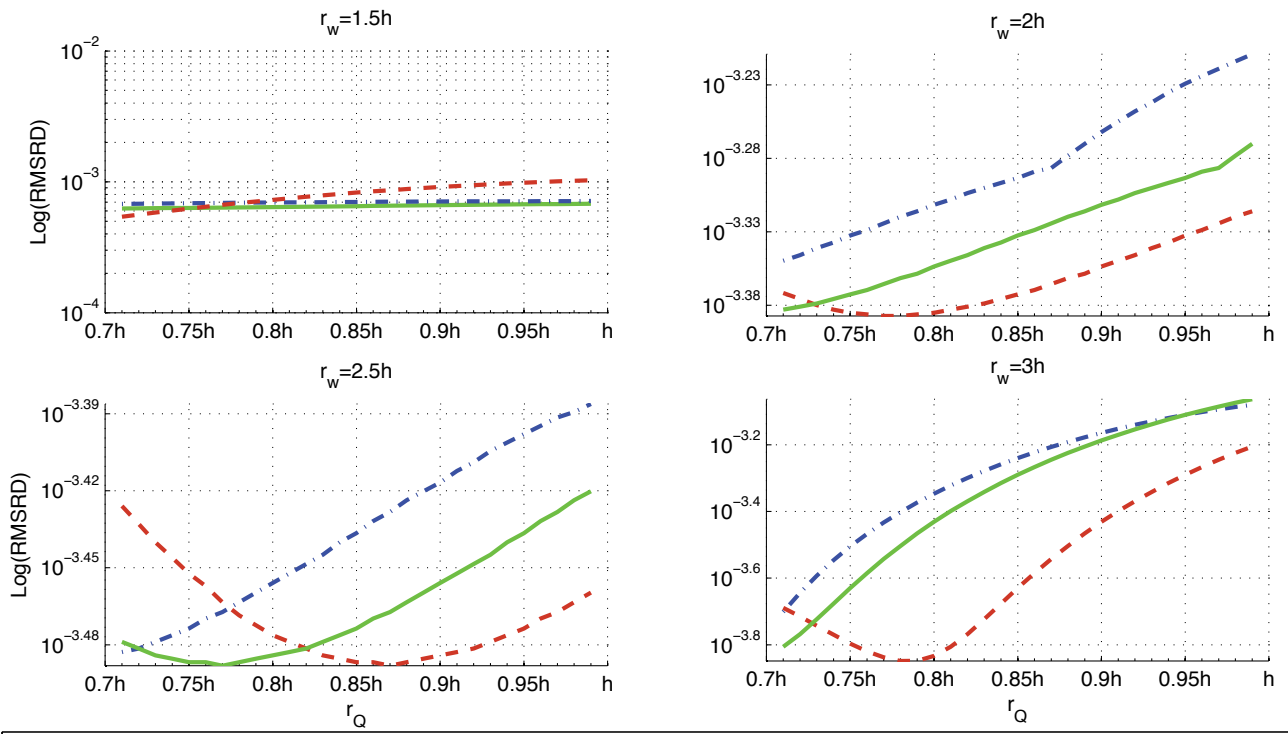

$=-=$ WCS-RBFs with $C^{6}$ smoothness degree $=1=$ ' WCS-RBFs with $C^{2}$ smoothness degree

wCS-RBFs with $\mathrm{C}^{4}$ smoothness degree

Figure 4: Test case 2: American call option under SVJ model using model parameters presented in [60, 11]. In these figures, the effect of the radius of the local sub-domains $r_{Q}$ and the size of the support domain $r_{w}$ on MaxError (or RMSRD) are shown to choose the optimal values of the radius of the local sub-domains. Here, we use $r_{w}=l h$, where $l=1.5,2,2.5,3$, and $h$ is the distance between the nodes. The radius of the local sub-domains $r_{Q}$ and the size of the support domain $r_{w}$ should be chosen to reduce the value of MaxError (or RMSRD). 
Table 16: Test case 3: European option.

\begin{tabular}{|c|c|c|c|c|c|c|c|c|c|}
\hline \multirow[b]{2}{*}{$\left(N_{x}, N_{z}, M\right)$} & \multicolumn{3}{|c|}{$C^{2}$} & \multicolumn{3}{|c|}{$C^{4}$} & \multicolumn{3}{|c|}{$C^{6}$} \\
\hline & RMSRD & Ratio & CPU time & RMSRD & Ratio & CPU time & RMSRD & Ratio & CPU time \\
\hline$(16,8,8)$ & $3.41 \mathrm{E}-02$ & - & 0.13 & $1.59 \mathrm{E}-02$ & - & 0.13 & $7.86 \mathrm{E}-03$ & - & 0.13 \\
\hline$(32,16,16)$ & $5.59 \mathrm{E}-03$ & 2.61 & 0.22 & $4.93 \mathrm{E}-03$ & 1.69 & 0.22 & $3.15 \mathrm{E}-03$ & 1.32 & 0.22 \\
\hline$(64,32,32)$ & $1.53 \mathrm{E}-03$ & 1.87 & 0.71 & $1.35 \mathrm{E}-03$ & 1.87 & 0.71 & $9.30 \mathrm{E}-04$ & 1.76 & 0.71 \\
\hline$(128,64,64)$ & 4.05E-04 & 1.92 & 1.81 & $3.68 \mathrm{E}-04$ & 1.88 & 1.81 & $2.58 \mathrm{E}-04$ & 1.85 & 1.81 \\
\hline$(256,128,128)$ & $1.42 \mathrm{E}-04$ & 1.51 & 2.49 & $9.66 \mathrm{E}-05$ & 1.93 & 2.49 & 7.73E-05 & 1.74 & 2.49 \\
\hline$(512,256,256)$ & $3.64 \mathrm{E}-05$ & 1.96 & 6.02 & $2.50 \mathrm{E}-05$ & 1.95 & 6.02 & $1.96 \mathrm{E}-05$ & 1.98 & 6.02 \\
\hline$(1024,512,512)$ & $9.30 \mathrm{E}-06$ & 1.97 & 24.53 & $6.42 \mathrm{E}-06$ & 1.96 & 24.53 & $4.86 \mathrm{E}-06$ & 2.01 & 24.53 \\
\hline$(2048,1024,1024)$ & $2.44 \mathrm{E}-06$ & 1.93 & 83.41 & $1.65 \mathrm{E}-06$ & 1.96 & 83.41 & $1.18 \mathrm{E}-06$ & 2.04 & 83.41 \\
\hline
\end{tabular}

Table 17: Test case 3: American option.

\begin{tabular}{|c|c|c|c|c|c|c|c|c|c|}
\hline \multirow[b]{2}{*}{$\left(N_{x}, N_{z}, M\right)$} & \multicolumn{3}{|c|}{$C^{2}$} & \multicolumn{3}{|c|}{$C^{4}$} & \multicolumn{3}{|c|}{$C^{6}$} \\
\hline & RMSRD & Ratio & CPU time & RMSRD & Ratio & CPU time & RMSRD & Ratio & CPU time \\
\hline$(16,8,8)$ & $1.51 \mathrm{E}-02$ & - & 0.13 & $1.63 \mathrm{E}-02$ & - & 0.20 & $1.11 \mathrm{E}-02$ & - & 0.20 \\
\hline$(32,16,16)$ & $2.66 \mathrm{E}-03$ & 2.51 & 0.22 & $2.40 \mathrm{E}-03$ & 2.76 & 0.38 & $2.41 \mathrm{E}-03$ & 2.21 & 0.38 \\
\hline$(64,32,32)$ & $6.97 \mathrm{E}-04$ & 1.93 & 0.71 & $6.31 \mathrm{E}-04$ & 1.93 & 1.01 & $8.89 \mathrm{E}-04$ & 1.44 & 1.01 \\
\hline$(128,64,64)$ & $2.97 \mathrm{E}-04$ & 1.23 & 1.81 & $2.08 \mathrm{E}-04$ & 1.60 & 3.62 & $2.50 \mathrm{E}-04$ & 1.83 & 3.62 \\
\hline$(256,128,128)$ & $8.65 \mathrm{E}-05$ & 1.78 & 2.49 & $6.72 \mathrm{E}-05$ & 1.63 & 4.17 & $7.71 \mathrm{E}-05$ & 1.70 & 4.17 \\
\hline$(512,256,256)$ & $2.59 \mathrm{E}-05$ & 1.74 & 6.02 & $2.14 \mathrm{E}-05$ & 1.65 & 10.96 & $2.08 \mathrm{E}-05$ & 1.89 & 10.96 \\
\hline$(1024,512,512)$ & $8.42 \mathrm{E}-06$ & 1.62 & 24.53 & $6.32 \mathrm{E}-06$ & 1.76 & 40.00 & $5.45 \mathrm{E}-06$ & 1.93 & 40.00 \\
\hline$(2048,1024,1024)$ & $2.47 \mathrm{E}-06$ & 1.77 & 83.41 & $1.79 \mathrm{E}-06$ & 1.82 & 137.21 & $1.42 \mathrm{E}-06$ & 1.94 & 137.21 \\
\hline
\end{tabular}

(3) As far as the time discretization is concerned, we used the implicit-explicit (IMEX) time stepping scheme. Note that, in stochastic volatility model with jumps, the integral part is a non-local integral, whereas the other parts which are differential operators, are all local. No doubt, since the integral part is non-local operator, a dense linear system of equations will be obtained by using the $\theta$-weighted discretization scheme. Therefore, to obtain a sparse linear system of equations, it is better to use an IMEX scheme which is noted for avoiding dense matrices. So far published work existing in the literature which use the IMEX scheme to price the options, include [57]. Such an approach is only first-order accurate, however a secondorder time discretization is obtained by performing a Richardson extrapolation procedure with halved time step.

(4) Up to now, only strong form meshless methods based on radial basis functions (RBFs) have been used for option pricing under SV model [10]. These techniques yield high levels of accuracy, but have of a very serious drawback such as produce a very ill-conditioned systems and very sensitive to the select of collocation points. Again, we do emphasize that in the new methods presented in this manuscript, coefficient matrix of the linear systems are sparse.

(5) LRPI scheme is the truly meshless methods, because, a traditional non-overlapping, continuous mesh is not required, neither for the construction of the shape functions, nor for the integration of the local sub-domains.

(6) Meshless methods using global RBFs such as Gaussian and multiquadric RBFs have a free parameter known as shape parameter. Despite many research works which are done to find algorithms for selecting the optimum values of $\epsilon[16,15,27,54,59]$, the optimal choice of shape parameter is an open problem which is still under intensive investigation. In general, as the value of the shape parameter $\epsilon$ decreases, the matrix of the system to be solved becomes highly ill-conditioned. To overcome this drawback of the global RBFs, the local RBFs such as Wendland compactly supported radial basis functions, which are local and stable functions, are proposed which are applied in this work.

(7) In LRPI method, using the delta Kronecker property, the boundary conditions can be easily imposed.

(8) The optimal values of the size of local sub-domain and support domain $\left(r_{Q}\right.$ and $\left.r_{w}\right)$ are illustrated using the Figures 3 and 4 for error vs. different values of $r_{Q}$ and $r_{w}$.

(9) Numerical experiments are presented showing that the LBIE and LRPI approaches are extremely accurate and fast.

(10) Future work will concern an extension to the basket options.

Acknowledgments The authors would like to thank anonymous reviewers for their careful reading of this manuscript and constructive comments which have helped improve the quality of the paper. The first authors work was supported by a Grant No. 94028928 sponsored by the Iran National Science Foundation (INSF). He sincerely thanks to INSF for their financial support.

\section{References}




\section{References}

[1] S. Abbasbandy, H. R. Ghehsareh, M. S. Alhuthali, H. H. Alsulami, Comparison of meshless local weak and strong forms based on particular solutions for a non-classical 2-D diffusion model, Eng. Anal. Bound. Elem. 39 (2014) 121-128.

[2] S. Abbasbandy, A. Shirzadi, MLPG method for two-dimensional diffusion equation with Neumann's and non-classical boundary conditions, Appl. Numer. Math. 61 (2011) 170-180.

[3] L. Andersen, J. Andreasen, Jump-diffusion processes: Volatility smile fitting and numerical methods for option pricing, Rev. Derivatives Res. 4 (2000) 231-262.

[4] P. Assari, H. Adibi, M. Dehghan, A meshless method for solving nonlinear two-dimensional integral equations of the second kind on non-rectangular domains using radial basis functions with error analysis, J. Comput. Appl. Math. 239 (2013) 72-92.

[5] P. Assari, H. Adibi, M. Dehghan, A meshless discrete galerkin (MDG) method for the numerical solution of integral equations with logarithmic kernels, J. Comput. Appl. Math. 267 (2014) 160-181.

[6] S. N. Atluri, S. Shen, Combination of meshless local weak and strong (MLWS) forms to solve the two dimensional hyperbolic telegraph equation, CMES 3 (2002) 11-51.

[7] S. N. Atluri, T. Zhu, A new meshless Local Petrov-Galerkin (MLPG) approach in computational mechanics, Comput. Mech. 22 (1998) 117-127.

[8] L. V. Ballestra, G. Pacelli, Computing the survival probability density function in jump-diffusion models: A new approach based on radial basis functions, Eng. Anal. Bound. Elem. 35 (2011) 10751084.

[9] L. V. Ballestra, G. Pacelli, A radial basis function approach to compute the first-passage probability density function in two-dimensional jump-diffusion models for financial and other applications, Eng. Anal. Bound. Elem. 36 (2012) 1546-1554.

[10] L. V. Ballestra, G. Pacelli, Pricing European and American options with two stochastic factors: A highly efficient radial basis function approach, J. Econ. Dyn. Cont. 37 (2013) 1142-1167.

[11] L. V. Ballestra, C. Sgarra, The evaluation of American options in a stochastic volatility model with jumps: An efficient finite element approach, Comput. Math. Appl. 60 (2010) 1571-1590.

[12] D. Bates, Jumps and stochastic volatility: the exchange rate processes implicit in Deutschemark options, Rev. Fin. Studies 9 (1996) 69-107.

[13] F. Black, M. Scholes, The pricing of options and corporate liabilities, J. Polit. Econ. 81 (1973) 637-659.

[14] M. D. Buhmann, Radial Basis Functions: Theory and Implementations, Cambridge University Press, New York, 2004.

[15] R. E. Carlson, T. A. Foley, The parameter $r^{2}$ in multiquadric interpolation, Comput. Math. Appl. 21 (1991) 29-42.

[16] A. H. D. Cheng, M. A. Golberg, E. J. Kansa, Q. Zammito, Exponential convergence and H-c multiquadric collocation method for partial differential equations, Numer. Meth. Part. D. E. 19 (2003) $571-594$.

[17] C. Chiarella, B. Kang, G. H. Meyer, A. Ziogas, The evaluation of American option prices under stochastic volatility and jump-diffusion dynamics using the method of lines, Int. J. Theor. Appl. Finance 12 (2009) 393-425.

[18] S. L. Chung, C. C. Chang, R. C. Stapleton, Richardson extrapolation techniques for the pricing of American-style options, J. Futures Markets 27 (2007) 791-817. 
[19] N. Clarke, K. Parrott, The multigrid solution of two-factor American put options, Technical Report 96-16, Oxford Comp. Lab, Oxford (1996).

[20] N. Clarke, K. Parrott, Multigrid for American option pricing with stochastic volatility, Appl. Math. Finance 6 (1999) 177-195.

[21] R. Cont, E. Voltchkova, A finite difference scheme for option pricing in jump diffusion and exponential levy models, SIAM J. Numer. Anal. 43 (2005) 1596-1626.

[22] M. Dehghan, A. Ghesmati, The Meshless Local Petrov-Galerkin (MLPG) method: A simple and lesscostly alternative to the finite element and boundary element methods, Eng. Anal. Bound. Elem. 34 (2010) 324-336.

[23] M. Dehghan, D. Mirzaei, The meshless local Petrov-Galerkin MLPG method for the generalized twodimensional non-linear Schrodinger equation, Eng. Anal. Bound. Elem. 32 (2008) 747-756.

[24] M. Dehghan, D. Mirzaei, Meshless local boundary integral equation (LBIE) method for the unsteady magnetohydrodynamic (MHD) flow in rectangular and circular pipes, Comput. Phys. Commun. 180 (2009) 1458-1466.

[25] M. Dehghan, D. Mirzaei, Meshless local Petrov-Galerkin (MLPG) method for the unsteady magnetohydrodynamic (MHD) flow through pipe with arbitrary wall conductivity, Appl. Numer. Math 59 (2009) 1043-1058.

[26] D. Duffie, J. Pan, K. Singleton, Transform analysis and asset pricing for affine jump-diffusions, Econometrica 68 (2000) 1343-1376.

[27] G. Fasshauer, J. Zhang, On choosing "optimal" shape parameters for RBF approximation, Numer. Algorithms 45 (2007) 346-368.

[28] L. Feng, V. Linetsky, Pricing options in jump-diffusion models: an extrapolation approach, Oper. Res. 56 (2008) 304-325.

[29] A. Golbabai, D. Ahmadian, M. Milev, Radial basis functions with application to finance: American put option under jump diffusion, Math. Comp. Modelling 55 (2012) 1354-1362.

[30] G. H. Golub, C. F. V. Loan, Matrix Computation, The Johns Hopkins University Press, 1989.

[31] S. L. Heston, A closed-form solution for options with stochastic volatility with applications to bond and currency options, The Review of Financial Studies 6 (1993) 327-343.

[32] Y. C. Hon, X. Mao, A radial basis function method for solving options pricing models, Financ. Eng. 8 (1999) 31-49.

[33] S. Ikonen, J. Toivanen, Efficient numerical methods for pricing American options under stochastic volatility, Numer. Meth. Partial Differ. Equ. 24 (2008) 104-126.

[34] S. Ikonen, J. Toivanen, Operator splitting methods for pricing American options under stochastic volatility, Numer. Math. 113 (2009) 299-324.

[35] K. Ito, J. Toivanen, Lagrange multiplier approach with optimized finite difference stencils for pricing American options under stochastic volatility, Siam J. Sci. Comput. 31 (2009) 2646-2664.

[36] A. Khaliq, G. Fasshauer, D. Voss, Using meshfree approximation for multi-asset American option problems, J. Chinese Institute Engineers 27 (2004) 563-571.

[37] S. G. Kou, A jump-diffusion model for option pricing, Manage. Sci. 48 (2002) 1086-1101.

[38] D. Krige, A review of the development of geostatistics in south africa, in: M. david, c. huijbregts (eds.), Advanced Geostatistics in the Mining Industry, Holland (1976) 279-293. 
[39] Y. Kwon, Y. Lee, A second-order finite difference method for option pricing under jump-diffusion models, SIAM J. Numer. Anal. 49 (2011) 2598-2617.

[40] Y. Kwon, Y. Lee, A second-order tridiagonal method for American options under jump-diffusion models, SIAM J. Sci. Comput. 33 (2011) 1860-1872.

[41] H. Lin, S. N. Atluri, Meshless local Petrov-Galerkin (MLPG) method for convection-diffusion problems, Comput. Model. Eng. Sci. 1 (2000) 45-60.

[42] G. Liu, Y. Gu, An Introduction to Meshfree Methods and Their Programing, Springer, Netherlands, 2005.

[43] M. D. Marcozzi, S. Choi, C. S. Chen, On the use of boundary conditions for variational formulations arising in financial mathematics, App. Math. Comput. 124 (2003) 197-214.

[44] R. C. Merton, Option pricing when underlying stock returns are discontinuous, J. Financ. Econ. 3 (1976) 125-144.

[45] E. Ngounda, K. C. Patidar, E. Pindza, A robust spectral method for solving Heston's model, J. Optim. Theory Appl. 161 (2014) 164-178.

[46] C. W. Oosterlee, On multigrid for linear complementarity problemswith application to American-style options, Electron. Trans. Numer. Anal. 15 (2003) 165-185.

[47] K. Parand, J. A. Rad, Numerical solution of nonlinear Volterra-Fredholm-Hammerstein integral equations via collocation method based on radial basis functions, App. Math. Comput. 218 (2012) 52925309.

[48] J. Persson, L. Sydow, Pricing American options using a space-time adaptive finite difference method, Math. Comput. Simul. 80 (2010) 1922-1935.

[49] J. Rad, K. Parand, Pricing American options under jump-diffusion models using local weak form meshless techniques, I. J. Comput. Math. dx.doi.org/10.1080/00207160.2016.1227434.

[50] J. A. Rad, S. Kazem, K. Parand, A numerical solution of the nonlinear controlled Duffing oscillator by radial basis functions, Comput. Math. Appl. 64 (2012) 2049-2065.

[51] J. A. Rad, K. Parand, S. Abbasbandy, Local weak form meshless techniques based on the radial point interpolation (RPI) method and local boundary integral equation (LBIE) method to evaluate European and American options, Commun. Nonlinear Sci. Numer. Simulat. 22 (2015) 1178-1200.

[52] J. A. Rad, K. Parand, S. Abbasbandy, Pricing European and American options using a very fast and accurate scheme-the meshless local Petrov-Galerkin (MLPG), Proc. Natl. Acad. Sci., India, Sect. A Phys. Sci. 85 (2015) 337-351.

[53] J. A. Rad, K. Parand, L. V. Ballestra, Pricing European and American options by radial basis point interpolation, Appl. Math. Comput. 251 (2015) 363-377.

[54] S. Rippa, An algorithm for selecting a good parameter $c$ in radial basis function interpolation, Advan. Comp. Math. 11 (1999) 193-210.

[55] S. Salmi, Numerical methods for pricing options under jump-diffusion processes, Ph.D. thesis, University of Jyvaskyla (2013).

[56] S. Salmi, J. Toivanen, An iterative method for pricing American options under jump-diffusion models, Appl. Numer. Math. 61 (2011) 821-831.

[57] S. Salmi, J. Toivanen, L. von Sydow, An IMEX-scheme for pricing options under stochastic volatility models with jumps, SIAM J. Sci. Comput. 36 (2014) 817-834. 
[58] A. Shirzadi, L. Ling, S. Abbasbandy, Meshless simulations of the two-dimensional fractional-time convection-diffusion-reaction equations, Eng. Anal. Bound. Elem. 36 (2012) 1522-1527.

[59] A. E. Tarwater, A parameter study of Hardy's multiquadric method for scattered data interpolation, Report UCRL-53670, Lawrence Livermore National Laboratory, 1985.

[60] J. Toivanen, A componentwise splitting method for pricing American options under the Bates model, in: W. Fitzgibbon, Yu. Kuznetsov, P. Neittaanmaki, J. Periaux, O. Pironneau (Eds.), Applied and Numerical Partial Differential Equations: Scientific Computing, Simulation, Optimization and Control in a Multidisciplinary Context, in: Computational Methods in Applied Sciences, 15, Springer, 2010, 213-227.

[61] H. Wendland, Scattered Data Approximation, Cambridge University Press, New York, 2005.

[62] P. Wilmott, J. Dewynne, S. Howison, Option Pricing: Mathematical Models and Computation, Oxford Financial Press, 1996.

[63] Z. Wu, Y. C. Hon, Convergence error estimate in solving free boundary diffusion problem by radial basis functions method, Eng. Anal. Bound. Elem. 27 (2003) 73-79.

[64] M. Yousuf, A. Q. M. Khaliq, B. Kleefeld, An efficient ETD method for pricing American options under stochastic volatility with nonsmooth payoffs, Numer. Meth. Partial Differential Eq. 29 (2013) $1864-1880$.

[65] S. P. Zhu, W. T. Chen, A predictor-corrector scheme based on the ADI method for pricing American puts with stochastic volatility, Comput. Math. Appl. 62 (2011) 1-26.

[66] R. Zvan, P. A. Forsyth, K. R. Vetzal, Penalty methods for American options with stochastic volatility, J. Comput. Appl. Math. 91 (1998) 199-218. 